\title{
Compositions of consistent systems of rank one discrete valuation rings
}

\author{
William J. Heinzer, Louis J. Ratliff, Jr., and David E. Rush
}

June 8, 2018

\begin{abstract}
Let $V$ be a rank one discrete valuation ring (DVR) on a field $F$ and let $L / F$ be a finite separable algebraic field extension with $[L: F]=m$. The integral closure of $V$ in $L$ is a Dedekind domain that encodes the following invariants: (i) the number $s$ of extensions of $V$ to a valuation ring $W_{i}$ on $L$, (ii) the residue degree $f_{i}$ of $W_{i}$ over $V$, and (iii) the ramification degree $e_{i}$ of $W_{i}$ over $V$. These invariants are related by the classical formula $m=\sum_{i=1}^{s} e_{i} f_{i}$. Given a finite set $\mathbf{V}$ of DVRs on the field $F$, an $m$-consistent system for $\mathbf{V}$ is a family of sets enumerating what is theoretically possible for the above invariants of each $V \in \mathbf{V}$. The $m$-consistent system is said to be realizable for $\mathbf{V}$ if there exists a finite separable extension field $L / F$ that gives for each $V \in \mathbf{V}$ the listed invariants. We investigate the realizability of $m$-consistent systems for $\mathbf{V}$ for various positive integers $m$. Our general technique is to "compose" several realizable consistent systems to obtain new consistent systems that are realizable for $\mathbf{V}$. We apply the new results to the set of Rees valuation rings of a nonzero proper ideal $I$ in a Noetherian domain $R$ of altitude one.
\end{abstract}

\section{Introduction.}

All rings in this paper are commutative with a unit $1 \neq 0$. Let $I$ be a regular proper ideal of the Noetherian ring $R$, that is, $I$ contains a regular element of $R$ and $I \neq R$. An ideal $J$ of $R$ is projectively equivalent to $I$ if there exist positive integers $m$ and $n$ such that $\left(I^{m}\right)_{a}=\left(J^{n}\right)_{a}$, where $K_{a}=\left\{x \in R \mid x\right.$ satisfies an equation of the form $x^{h}+k_{1} x^{h-1}+\cdots+k_{h}$, where $k_{j} \in K^{j}$ for $\left.j=1, \ldots, k\right\}$ is the integral closure in $R$ of an ideal $K$ of $R$. The concept of projective equivalence of ideals and the study of ideals projectively equivalent to $I$ was introduced by Samuel in [16] and further developed by Nagata in [13] and Rees in [14]. See [4] for a recent survey. Let $\mathbf{P}(I)$ denote the set of integrally closed ideals that are projectively equivalent to $I$. The ideal $I$ is said to be projectively full if $\mathbf{P}(I)$ 
$=\left\{\left(I^{n}\right)_{a} \mid n \geq 1\right\}$ and $\mathbf{P}(I)$ is said to be projectively full if $\mathbf{P}(I)=\mathbf{P}(J)$ for some projectively full ideal $J$ of $R$.

The set Rees $I$ of Rees valuation rings of $I$ is a finite set of rank one discrete valuation rings (DVRs) that determine the integral closure $\left(I^{n}\right)_{a}$ of $I^{n}$ for every positive integer $n$ and are the unique minimal set of DVRs having this property. Consider the minimal primes $z$ of $R$ such that $I R / z$ is a proper nonzero ideal. The set Rees $I$ is the union of the sets Rees $I R / z$. Thus one is reduced to describing the set Rees $I$ in the case where $I$ is a nonzero proper ideal of a Noetherian integral domain $R$. Consider the Rees ring $\mathbf{R}=R\left[t^{-1}, I t\right]$. The integral closure $\mathbf{R}^{\prime}$ of $\mathbf{R}$ is a Krull domain, so $W=\mathbf{R}_{p}^{\prime}$ is a DVR for each minimal prime $p$ of $t^{-1} \mathbf{R}^{\prime}$, and $V=W \cap F$, where $F$ is the field of fractions of $R$, is also a DVR. The set Rees $I$ of Rees valuation rings of $I$ is the set of DVRs $V$ obtained in this way, cf. [17, Section 10.1].

If $\left(V_{1}, N_{1}\right), \ldots,\left(V_{n}, N_{n}\right)$ are the Rees valuation rings of $I$, then the integers $\left(e_{1}, \ldots, e_{n}\right)$, where $I V_{i}=N_{i}^{e_{i}}$, are the Rees integers of $I$. Necessary and sufficient conditions for two regular proper ideals $I$ and $J$ to be projectively equivalent are that (i) Rees $I=$ Rees $J$ and (ii) the Rees integers of $I$ and $J$ are proportional [1, Theorem 3.4]. If $I$ is integrally closed and each Rees integer of $I$ is one, then $I$ is a projectively full radical ideal 1

A main goal in the papers [1], [2], [3], [4], [9] and [10], is to answer the following question:

Question 1.1 Let $I$ be a nonzero proper ideal in a Noetherian domain $R$. Under what conditions does there exist a finite integral extension domain $A$ of $R$ such that $\mathbf{P}(I A)$ contains an ideal $J$ whose Rees integers are all equal to one?

Progress is made on Question 1.1 in [3]. To describe this progress, let $I$ be a regular proper ideal of the Noetherian $\operatorname{ring} R$, let $b_{1}, \ldots, b_{g}$ be regular elements in $R$ that generate $I$, and for each positive integer $m>1$ let $A_{m}=R\left[x_{1}, \ldots, x_{g}\right]=R\left[X_{1}, \ldots, X_{g}\right] /\left(X_{1}{ }^{m}-\right.$ $\left.b_{1}, \ldots, X_{g}{ }^{m}-b_{g}\right)$ and let $J_{m}=\left(x_{1}, \ldots, x_{g}\right) A_{m}$. Let $\left(V_{1}, N_{1}\right), \ldots,\left(V_{n}, N_{n}\right)$ be the Rees valuation rings of $I$. Consider the following hypothesis on $I=\left(b_{1}, \ldots, b_{g}\right) R$ :

(a) $b_{i} V_{j}=I V_{j}\left(=N_{j}{ }^{e_{j}}\right.$, say) for $i=1, \ldots, g$ and $j=1, \ldots, n$.

\footnotetext{
${ }^{1}$ Example 5.1 of [2] demonstrates that there exist integrally closed local domains $(R, M)$ for which $M$ is not projectively full. Remark 4.10 and Example 4.14 of [1] show that a sufficient, but not necessary, condition for $I$ to be projectively full is that the gcd of the Rees integers of $I$ is equal to one.
} 
(b) the greatest common divisor $c$ of $e_{1}, \ldots, e_{n}$ is a unit in $R$.

$\left(\mathrm{b}^{\prime}\right)$ the least common multiple $d$ of $e_{1}, \ldots, e_{n}$ is a unit in $R$.

Then the main result in [3] establishes the following:

Theorem 1.2 If (a) and (b) hold, then $A_{c}=R\left[x_{1}, \ldots, x_{g}\right]$ is a finite free integral extension ring of $R$ and the ideal $J_{c}=\left(x_{1}, \ldots, x_{g}\right) A_{c}$ is projectively full and projectively equivalent to $I A_{c}$. Also, if $R$ is an integral domain and if $z$ is a minimal prime ideal in $A_{c}$, then $\left(\left(J_{c}+z\right) / z\right)_{a}$ is a projectively full ideal in $A_{c} / z$ that is projectively equivalent to $\left(I A_{c}+z\right) / z$.

We prove in [9, (3.19) and (3.20)] that if either (i) $R$ contains an infinite field, or (ii) $R$ is a local ring with an infinite residue field, then it is possible to choose generators $b_{1}, \ldots, b_{g}$ of I that satisfy assumption (a) of Theorem [1.2. Thus the following result, [9, (3.7)] , applies in these cases.

Theorem 1.3 If (a) and ( $\left.\mathrm{b}^{\prime}\right)$ hold, then for each positive multiple $m$ of $d$ that is a unit in $R$ the ideal $\left(J_{m}\right)_{a}$ is projectively full and $\left(J_{m}\right)_{a}$ is a radical ideal that is projectively equivalent to $I A_{m}$. Also, the Rees integers of $J_{m}$ are all equal to one and $x_{i} U$ is the maximal ideal of $U$ for each Rees valuation ring $U$ of $J_{m}$ and for $i=1, \ldots, g$. Moreover, if $R$ is an integral domain and if $z$ is a minimal prime ideal in $A_{m}$, then $\left(\left(J_{m}+z\right) / z\right)_{a}$ is a projectively full radical ideal that is projectively equivalent to $\left(I A_{m}+z\right) / z$.

Examples [9, (3.22) and (3.23)] show that even if $R$ is the ring $\mathbb{Z}$ of rational integers, condition $\left(b^{\prime}\right)$ of Theorem 1.3 is needed for the proof given in [9]. Theorem 1.4 is the main result in $[10$.

Theorem 1.4 Let I be a nonzero proper ideal in a Noetherian integral domain $R$.

1. There exists a finite separable integral extension domain $A$ of $R$ and a positive integer $m$ such that all the Rees integers of IA are equal to $m$.

2. If $R$ has altitude one, then there exists a finite separable integral extension domain $A$ of $R$ such that $\mathbf{P}(I A)$ contains an ideal $H$ whose Rees integers are all equal to 
one. Therefore $H=\operatorname{Rad}(I A)$ is a projectively full radical ideal that is projectively equivalent to IA.

Observe that Theorem 1.4.2, answers Question 1.1 in the affirmative for each nonzero proper ideal $I$ in an arbitrary Noetherian integral domain $R$ of altitude one with no additional conditions; therefore the conclusions of Theorems 1.2 and 1.3 are valid without the assumption of conditions (a), (b), and ( $\left.b^{\prime}\right)$ if $R$ is a Noetherian integral domain of altitude one. In particular, Theorem 1.4,2 shows that these conclusions hold for the examples [9, (3.22) and (3.23)].

A classical theorem of Krull, stated as Theorem 2.1 below, is an important tool in the present paper and in [10]. We use the following terminology from [7] and [10].

Definition 1.5 Let $\left(V_{1}, N_{1}\right), \ldots,\left(V_{n}, N_{n}\right)$ be distinct DVRs of a field $F$ and for $i=1, \ldots, n$ let $K_{i}=V_{i} / N_{i}$ denote the residue field of $V_{i}$. Let $m$ be a positive integer. By an $m$ consistent system for $\left\{V_{1}, \ldots, V_{n}\right\}$, we mean a collection of sets $S=\left\{S\left(V_{1}\right), \ldots, S\left(V_{n}\right)\right\}$ satisfying the following conditions:

(1) $S\left(V_{i}\right)=\left\{\left(K_{i, j}, f_{i, j}, e_{i, j}\right) \mid j=1, \ldots, s_{i}\right\}$, where $K_{i, j}$ is a simple algebraic field extension of $K_{i}, f_{i, j}=\left[K_{i, j}: K_{i}\right]$, and $s_{i}, e_{i, j} \in \mathbb{N}_{+}$(the set of positive integers).

(2) For each $i$, the sum $\sum_{j=1}^{s_{i}} e_{i, j} f_{i, j}=m$.

Definition 1.6 The $m$-consistent system $S$ for $\left\{V_{1}, \ldots, V_{n}\right\}$ as in Definition 1.5 is said to be realizable for $\left\{V_{1}, \ldots, V_{n}\right\}$ if there exists a separable algebraic extension field $L$ of $F$ such that:

(a) $[L: F]=m$.

(b) For $1 \leq i \leq n, V_{i}$ has exactly $s_{i}$ extensions $\left(V_{i, 1}, N_{i, 1}\right), \ldots,\left(V_{i, s_{i}}, N_{i, s_{i}}\right)$ to $L$.

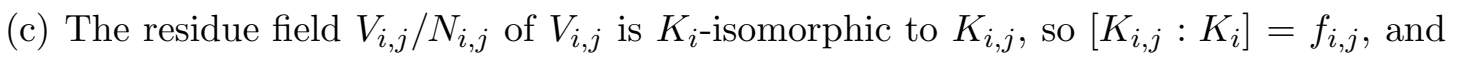
the ramification index of $V_{i, j}$ over $V_{i}$ is $e_{i, j}$, so $N_{i} V_{i, j}=N_{i, j}{ }^{e_{i, j}}$.

If $S$ and $L$ are as above, we say the field $L$ realizes $S$ for $\left\{V_{1}, \ldots, V_{n}\right\}$ or that $L$ is a realization of $S$ for $\left\{V_{1}, \ldots, V_{n}\right\}$.

Let $\mathbf{V}=\left\{V_{1}, \ldots, V_{n}\right\}, n>1$, be a finite set of distinct DVRs on the field $F$. In this paper we explore various facets of the realizability of consistent systems for $\mathbf{V}$. If 
$S=\left\{S\left(V_{1}\right), \ldots, S\left(V_{n}\right)\right\}$ is an $m$-consistent system for $\mathbf{V}$, realizable or not, we prove in Theorem 2.3 (resp., Theorem 2.7) that by uniformly increasing the ramification indices (resp., finite-residue-field degrees) by the factor $m$ the resulting system is a realizable $m^{2}$ consistent system for $\mathbf{V}$. The proofs involve composing two related realizable consistent systems.

Let $M_{1}, \ldots, M_{n}, n>1$, be distinct maximal ideals of the Dedekind domain $D$ and let $\mathbf{V}=\left\{D_{M_{1}}, \ldots, D_{M_{n}}\right\}$ be the related family of DVRs. Let $I=M_{1}{ }^{e_{1}} \cdots M_{n}{ }^{e_{n}}$. Then by composing two related systems we prove in Theorem 3.3 that the $e_{1} \cdots e_{n}$-consistent system $S=\left\{S\left(D_{M_{1}}\right), \ldots, S\left(D_{M_{n}}\right)\right\}$ is realizable for $\mathbf{V}$, where $S\left(D_{M_{i}}\right)=\left\{\left(K_{i, j}, 1, \frac{e_{1} \cdots e_{n}}{e_{i}}\right) \mid j=\right.$ $\left.1, \ldots, e_{i}\right\}$ for $i=1, \ldots, n$. It follows that if $E$ is the integral closure of $D$ in a realization $L$ of $S$ for $\mathbf{V}$, and if $N_{i, 1}, \ldots, N_{i, e_{i}}$ are the maximal ideals in $E$ that contain $M_{i} E$, then $E / N_{i, j} \cong D / M_{i}$ and $M_{i} E_{N_{i, j}}=N_{i, j}^{\frac{e_{1} \cdots e_{n}}{e_{i}}}$, so $I E=(\operatorname{Rad}(I E))^{e_{1} \cdots e_{n}}$ is a radical power ideal in the sense that it is a power of its radical. We also prove a theorem analogous to Theorem 3.3 for each nonzero proper ideal in an arbitrary Noetherian domain of altitude one. Proposition 3.13 characterizes the conditions a realizable $m$-consistent system $S^{\prime}$ for $\mathbf{V}$ must satisfy to insure that $I E=(\operatorname{Rad}(I E))^{t}$ for some positive integer $t$, where $E$ is the integral closure of $D$ in a realization $L$ of $S^{\prime}$ for $\mathbf{V}$.

Under the hypothesis that each residue field $D / M_{i}$ is finite, we prove in Section 4 that every consistent system $T=\left\{T\left(D_{M_{1}}\right), \ldots, T\left(D_{M_{n}}\right)\right\}$ of the following form is realizable for $\mathbf{V}: T\left(D_{M_{i}}\right)=\left\{\left(K_{i, j}, \frac{f_{1} \cdots f_{n}}{f_{i}}, 1\right) \mid j=1, \ldots, f_{i}\right\}$ for $i=1, \ldots, n$; here the $f_{i}$ are arbitrary positive integers for which $\left[D / M_{i}: P_{i}\right]=f_{i}$ for some subfield $F_{i}$ of $D / M_{i}$. Therefore if $E$ is the integral closure of $D$ in a realization $L$ of $T$ for $\mathbf{V}$ and if $N_{i, 1}, \ldots, N_{i, f_{i}}$ are the maximal ideals in $E$ that contain $M_{i}$, then $\left[\left(E / N_{i, j}\right): F_{i}\right]=f_{1} \cdots f_{n}$ and $M_{i} E_{N_{i, j}}=N_{i, j} E_{N_{i, j}}$ for each $i=1, \ldots, n$ and each $j=1, \ldots, f_{i}$. Under the same hypothesis on the $D / M_{i}$, we establish in Section 4 finite-residue-field degree analogs of some of the other results in Section 3.

Under the hypothesis that each residue field $D / M_{i}$ is finite, we prove in Section 5 that every consistent system $U=\left\{U\left(D_{M_{1}}\right), \ldots, U\left(D_{M_{n}}\right)\right\}$ of the following form is realizable for $\mathbf{V}: U\left(D_{M_{i}}\right)=\left\{\left(K_{i, j}, \frac{f_{1} \cdots f_{n}}{f_{i}}, \frac{e_{1} \cdots e_{n}}{e_{i}}\right) \mid j=1, \ldots, e_{i} f_{i}\right\}$ for $i=1, \ldots, n$, where the $e_{i}$ and $f_{i}$ are as in Sections 3 and 4, respectively. Therefore if $E$ is the integral closure of $D$ in a realization $L$ of $U$ for $\mathbf{V}$ and if $N_{i, j}, \ldots, N_{i, e_{i} f_{i}}$ are the maximal ideals in $E$ that contain $M_{i}$, 
then $I E=(\operatorname{Rad}(I E))^{e_{1} \cdots e_{n}}$ and $\left[\left(E / N_{i, j}\right): F_{i}\right]=f_{1} \cdots f_{n}$ for each $i=1, \ldots, n$ and each $j$ $=1, \ldots, e_{i} f_{i}$.

Our notation is mainly as in Nagata [12, so, for example, the term altitude refers to what is often also called dimension or Krull dimension, and a basis of an ideal is a set of generators of the ideal.

\section{The realizability of $m$-consistent systems.}

To prove the main results in this section, we use the following theorem of Krull.

Theorem 2.1 (Krull [11]): Let $\left(V_{1}, N_{1}\right), \ldots,\left(V_{n}, N_{n}\right)$ be distinct DVRs with quotient field $F$, let $m$ be a positive integer, and let $S=\left\{S\left(V_{1}\right), \ldots, S\left(V_{n}\right)\right\}$ be an $m$-consistent system for $\left\{V_{1}, \ldots, V_{n}\right\}$, where $S\left(V_{i}\right)=\left\{\left(K_{i, j}, f_{i, j}, e_{i, j}\right) \mid j=1, \ldots, s_{i}\right\}$ for $i=1, \ldots, n$. Then $S$ is realizable for $\left\{V_{1}, \ldots, V_{n}\right\}$ if one of the following conditions is satisfied:

(i) $s_{i}=1$ for at least one $i$.

(ii) F has at least one $D V R V$ distinct from $V_{1}, \ldots, V_{n}$.

(iii) For each monic polynomial $X^{t}+a_{1} X^{t-1}+\cdots+a_{t}$ with $a_{i} \in \cap_{i=1}^{n} V_{i}=D$, and for each $h \in \mathbb{N}$, there exists an irreducible separable polynomial $X^{t}+b_{1} X^{t-1}+\cdots+b_{t} \in D[X]$ with $b_{l}-a_{l} \in N_{i}^{h}$ for each $l=1, \ldots, t$ and $i=1, \ldots, n$.

Observe that condition (i) of Theorem 2.1 is a property of the $m$-consistent system $S=$ $\left\{S\left(V_{1}\right), \ldots, S\left(V_{n}\right)\right\}$, whereas condition (ii) is a property of the family of DVRs with quotient field $F$, and condition (iii) is a property of the family $\left(V_{1}, N_{1}\right), \ldots,\left(V_{n}, N_{n}\right)$.

The result of Krull stated in Theorem 2.1 is a generalization of a classical result of Hasse [8] which shows that all $m$-consistent systems for a given finite set of distinct DVRs of an algebraic number field $F$ are realizable. This has been extended further by P. Ribenboim, O. Endler and L. C. Hill, among others. For a good sampling of these results on when an $m$-consistent system is realizable, see [5, Sections $25-27]$ and [6]. These references give several sufficient conditions on the realizability of an $m$-consistent system for a given finite set $\mathbf{V}=\left\{V_{1}, \ldots, V_{n}\right\}$ of distinct DVRs $V_{i}$ with quotient field $F$. 
Remark $2.2(\mathbf{2 . 2}, \mathbf{1})$ There is an obvious necessary condition for an $m$-consistent system to be realizable. If $n=1$ and $V_{1}$ is a Henselian DVR, then no $m$-consistent system $S=$ $\left\{S\left(V_{1}\right)\right\}$, where $S\left(V_{1}\right)=\left\{\left(K_{1}, f_{1}, e_{1}\right), \ldots,\left(K_{s}, f_{s}, e_{s}\right)\right\}$ with $s>1$ is realizable for $\left\{V_{1}\right\}$, since $V_{1}$ is Henselian if and only if $V_{1}$ has a unique extension to each finite algebraic extension field of its quotient field $F$, cf. [12, (43.12)]. It follows from Theorem 2.1(ii) that if $V$ is a Henselian DVR, then $V$ is the unique DVR with quotient field $F$. It is not true, however, that $V$ being the unique DVR on its quotient field implies that $V$ is Henselian. For example, using that the field $\mathbb{Q}$ of rational numbers admits only countably many DVRs, it is possible to repeatedly use Theorem 2.1 to construct an infinite algebraic extension field $F$ of $\mathbb{Q}$ such that $F$ admits a unique DVR $V$ having quotient field $F$ and yet $V$ is not Henselian.

$(\mathbf{2 . 2}, \mathbf{2})$ Related to $(2.2,1)$, it is shown in [15, Theorem 1] that, for each positive integer $n$, there exist fields $F_{n}$ that admit exactly $n$ DVRs $\left(V_{1}, N_{1}\right), \ldots,\left(V_{n}, N_{n}\right)$ having quotient field $F_{n}$. Moreover, the proof of [15, Theorem 1] shows that such $F_{n}$ can be chosen so that there are no realizable $m$-consistent systems $S$ for $\left\{V_{1}, \ldots, V_{n}\right\}$ having the property that $m>1$, and, for each $i=1, \ldots, n, S\left(V_{i}\right)=\left\{\left(K_{i, j}, f_{i, j}, e_{i, j}\right) \mid j=1, \ldots, s_{i}\right\}$ has at least one $j$ with $\left(K_{i, j}, f_{i, j}, e_{i, j}\right)=\left(V_{i} / N_{i}, 1,1\right)$.

Theorem 2.3, is a new sufficient condition for realizability; by Remark 2.2.1, the hypothesis $n>1$ in Theorem 2.3 is essential.

Theorem 2.3 Let $\left(V_{1}, N_{1}\right), \ldots,\left(V_{n}, N_{n}\right), n>1$, be distinct DVRs with quotient field $F$, let $m>1$ be a positive integer, and let $S=\left\{S\left(V_{1}\right), \ldots, S\left(V_{n}\right)\right\}$ be an arbitrary $m$-consistent system for $\left\{V_{1}, \ldots, V_{n}\right\}$, where, $S\left(V_{i}\right)=\left\{\left(K_{i, j}, f_{i, j}, e_{i, j}\right) \mid j=1, \ldots, s_{i}\right\}$, for each $i=1, \ldots, n$. Then $S^{*}=\left\{S^{*}\left(V_{1}\right), \ldots, S^{*}\left(V_{n}\right)\right\}$ is a realizable $m^{2}$-consistent system for $\left\{V_{1}, \ldots, V_{n}\right\}$, where $S^{*}\left(V_{i}\right)=\left\{\left(K_{i, j}, f_{i, j}, m e_{i, j}\right) \mid j=1, \ldots, s_{i}\right\}$, for each $i=1, \ldots, n$.

Proof. If $s_{i}=1$ for some $i=1, \ldots, n$, then Theorem 2.1(i) implies that $S$ is a realizable $m$-consistent system and $S^{*}$ is a realizable $m^{2}$-consistent system for $\left\{V_{1}, \ldots, V_{n}\right\}$, so it may be assumed that $s_{i}>1$ for each $i=1, \ldots, n$.

Define $S_{1}\left(V_{i}\right)=S\left(V_{i}\right)$ for $i=1, \ldots, n-1$ and $S_{1}\left(V_{n}\right)=\left\{\left(\left(V_{n} / N_{n}\right), 1, m\right)\right\}$, and recall that $n>1$. Theorem 2.1(i) implies that $S_{1}=\left\{S_{1}\left(V_{1}\right), \ldots, S_{1}\left(V_{n-1}\right), S_{1}\left(V_{n}\right)\right\}$ is a realizable 
$m$-consistent system for $\left\{V_{1}, \ldots, V_{n}\right\}$. Let $L_{1}$ be a realization of $S_{1}$ for $\left\{V_{1}, \ldots, V_{n}\right\}$. Thus $L_{1}$ is a separable algebraic extension field of $F$ of degree $m$. For $i=1, \ldots, n$ let $\left(W_{i, j}, N_{i, j}\right)$ be the valuation rings of $L_{1}$ that lie over $V_{i}$. It follows from the prescription of $S_{1}$ that there are exactly $s_{i}$ such rings for $i=1, \ldots, n-1$ and exactly one such ring for $i=n$. Also, $W_{i, j} / N_{i, j}$ is $\left(V_{i} / N_{i}\right)$-isomorphic to $K_{i, j}$ and $N_{i} W_{i, j}=N_{i, j} e_{i, j}$ for $i=1, \ldots, n-1$ and $j=$ $1, \ldots, s_{i}$, while $W_{n, 1} / N_{n, 1}$ is $\left(V_{n} / N_{n}\right)$-isomorphic to $V_{n} / N_{n}$ and $N_{n} W_{n, 1}=N_{n, 1}{ }^{m}$.

Let $S_{2}=\left\{S_{2}\left(W_{1,1}\right), \ldots, S_{2}\left(W_{n-1, s_{n-1}}\right), S_{2}\left(W_{n, 1}\right)\right\}$, where $S_{2}\left(W_{i, j}\right)=\left\{\left(K_{i, j}, 1, m\right)\right\}$ for $i=1, \ldots, n-1$ and $j=1, \ldots, s_{i}$, and where $S_{2}\left(W_{n, 1}\right)=\left\{\left(K_{n, j}, f_{n, j}, e_{n, j}\right) \mid j=1, \ldots, s_{n}\right\}$. Thus $S_{2}\left(W_{n, 1}\right)$ is essentially equal to $S\left(V_{n}\right)$. It is readily checked that $S_{2}$ is an $m$-consistent system for $\mathcal{W}:=\left\{W_{1,1}, \ldots, W_{n-1, s_{n-1}}, W_{n, 1}\right\}$, and by Theorem 2.1(i) it is realizable for $\mathcal{W}$. Let $L$ be a realization of $S_{2}$ for $\mathcal{W}$. Thus $L$ is a separable algebraic extension field of $L_{1}$ of degree $m$, and hence a separable algebraic extension field of $F$ of degree $m^{2}$. Moreover, for $i=1, \ldots, n-1$ and $j=1, \ldots, s_{i}$ there exists a unique valuation $\operatorname{ring}\left(U_{i, j}, P_{i, j}\right)$ of $L$ that lies over $W_{i, j}$, and $U_{i, j} / P_{i, j}$ is $\left(W_{i, j} / N_{i, j}\right)$-isomorphic to $W_{i, j} / N_{i, j}$; also, $W_{i, j} / N_{i, j}$ is $\left(V_{i} / N_{i}\right)$ isomorphic to $K_{i, j}$, so $U_{i, j} / P_{i, j}$ is $\left(V_{i} / N_{i}\right)$-isomorphic to $K_{i, j}$, and $N_{i, j} U_{i, j}=P_{i, j}{ }^{m}$, so $N_{i} U_{i, j}$ $=N_{i, j} e_{i, j}$. On the other hand, for $i=n$ there are exactly $s_{n}$ valuation $\operatorname{rings}\left(U_{n, j}, P_{n, j}\right)$ that lie over $\left(W_{n, 1}, N_{n, 1}\right)$, and for $j=1, \ldots, s_{n}, U_{n, j} / P_{n, j}$ is $\left(W_{n, 1} / N_{n, 1}\right)$-isomorphic to $K_{n, j}$, and $W_{n, 1} / N_{n, 1}$ is $\left(V_{n} / N_{n}\right)$-isomorphic to $V_{n} / N_{n}$, so $U_{n, j} / P_{n, j}$ is $\left(V_{n} / N_{n}\right)$-isomorphic to $K_{n, j}$, and $N_{n, 1} U_{n, j}=P_{n, j} e_{n, j}$, so $N_{n} U_{n, j}=P_{n, j} e_{n, j}$. It therefore follows that $L$ is a realization of the $m^{2}$-consistent system $S^{*}=\left\{S^{*}\left(V_{1}\right), \ldots, S^{*}\left(V_{n}\right)\right\}$ for $\left\{V_{1}, \ldots, V_{n}\right\}$, where $S^{*}\left(V_{i}\right)=$ $\left\{\left(K_{i, j}, f_{i, j}, m e_{i, j}\right) \mid j=1, \ldots, s_{i}\right\}$ for $i=1, \ldots, n$. Thus $S^{*}$ is a realizable $m^{2}$-consistent system for $\left\{V_{1}, \ldots, V_{n}\right\}$.

Remark 2.4 Fix $g \in\{1, \ldots, n-1\}$. Then essentially the same proof as given for Theorem 2.3 shows that the following two $m$-consistent systems $T_{1}, T_{2}$ are realizable and can be used in place of $S_{1}, S_{2}$ to prove Theorem 2.3. $T_{1}=\left\{T_{1}\left(V_{1}\right), \ldots, T_{1}\left(V_{n}\right)\right\}$, where $T_{1}\left(V_{i}\right)$ $=S\left(V_{i}\right)$ for $i=1, \ldots, g$, while $T_{1}\left(V_{h}\right)=\left\{\left(\left(V_{h} / N_{h}\right), 1, m\right)\right\}$ for $h=g+1, \ldots, n$. $T_{2}=$ $\left\{T_{2}\left(W_{1,1}\right), \ldots, T_{2}\left(W_{g, s_{g}}\right), T_{2}\left(W_{g+1,1}\right), \ldots, T_{2}\left(W_{n, 1}\right)\right\}$, where $T_{2}\left(W_{i, j}\right)=\left\{\left(K_{i, j}, 1, m\right)\right\}$ for $i$ $=1, \ldots, g$ and $j=1, \ldots, s_{i}$, while $T_{2}\left(W_{h, 1}\right)=\left\{\left(K_{h, j}, f_{h, j}, e_{h, j}\right) \mid j=1, \ldots, s_{h}\right\}$ for $h=$ $g+1, \ldots, n$ (so $T_{2}\left(W_{h, 1}\right)$ is essentially equal to $T\left(V_{h}\right)$ for $h=g+1, \ldots, n$ ). 
Corollary 2.5 Let $R$ be a Noetherian domain, let $I$ be a nonzero proper ideal in $R$, let $\left(V_{1}, N_{1}\right), \ldots,\left(V_{n}, N_{n}\right)$ be the Rees valuation rings of $I$, let $m, s_{1}, \ldots, s_{n}$ be positive integers, and let $S=\left\{S\left(V_{1}\right), \ldots, S\left(V_{n}\right)\right\}$ be an arbitrary $m$-consistent system for $\left\{V_{1}, \ldots, V_{n}\right\}$, say $S\left(V_{i}\right)=\left\{\left(K_{i, j}, f_{i, j}, e_{i, j}\right) \mid j=1, \ldots, s_{i}\right\}$ for $i=1, \ldots, n$. Then there exists a separable algebraic extension field $L$ of degree $m^{2}$ of the quotient field $R_{(0)}$ of $R$ such that, for each finite integral extension domain $A$ of $R$ with quotient field $L$ and for $i=1, \ldots, n, I A$ has exactly $s_{i}$ Rees valuation rings $\left(W_{i, j}, N_{i, j}\right)$ that extend $\left(V_{i}, N_{i}\right)$, and then, for $j=1, \ldots, s_{i}$, the Rees integer of $I A$ with respect to $W_{i, j}$ is $m e_{i, j}$ and $\left[\left(W_{i, j} / N_{i, j}\right):\left(V_{i} / N_{i}\right)\right]=f_{i, j}$.

Proof. By [10, Remark 2.7] the extensions of the Rees valuation rings of $I$ to the field $L$ are the Rees valuation rings of $I A$, so this follows immediately from Theorem 2.3 ,

Theorem 2.7, is a new sufficient condition for realizability under the hypothesis that each of the valuation rings $\left(V_{i}, N_{i}\right), 1 \leq i \leq n$, has a finite residue field. For this result and the results in Sections 4 and 5 we often implicitly use the following remark.

Remark 2.6 (2.6.1) Let $F$ be a finite field. It is well known, see for example [19, pages 8284], that the following hold: (i) Each finite extension field $H$ of $F$ is separable and thus a simple extension of $F$. (ii) If $k$ is a positive integer and $\bar{F}$ is a fixed algebraic closure of $F$, then there exists a unique extension field $H \subseteq \bar{F}$ with $[H: F]=k$. (iii) If $H, K \subseteq \bar{F}$ are finite extension fields of $F$, then $H \subseteq K$ if and only if $[H: F]$ divides $[K: F]$.

(2.6,2) There are fields other than finite fields that satisfy the three conditions given in (2.6.1). If $E$ is an algebraically closed field of characteristic zero and $F$ is the field of fractions of the formal power series ring $E[[x]]$, then a theorem that goes back to Newton implies that $F$ satisfies the conditions of [2.6.1) cf. [18, Theorem 3.1, page 98].

Theorem 2.7 Let $\left(V_{1}, N_{1}\right), \ldots,\left(V_{n}, N_{n}\right)(n>1)$ be distinct DVRs with quotient field $F$, where each $V_{i} / N_{i}$ is finite. For each $i$ let $\overline{V_{i} / N_{i}}$ denote a fixed algebraic closure of $V_{i} / N_{i}$. Let $m$ be a positive integer, and let $S=\left\{S\left(V_{1}\right), \ldots, S\left(V_{n}\right)\right\}$ be an arbitrary $m$-consistent system for $\left\{V_{1}, \ldots, V_{n}\right\}$, where, for $i=1, \ldots, n, S\left(V_{i}\right)=\left\{\left(K_{i, j}, f_{i, j}, e_{i, j}\right) \mid K_{i, j} \subseteq \overline{V_{i} / N_{i}}\right.$ and $\left.j=1, \ldots, s_{i}\right\}$. For $i=1, \ldots, n$ let $T^{*}\left(V_{i}\right)=\left\{\left(K_{i, j}{ }^{*}, m f_{i, j}, e_{i, j}\right) \mid j=1, \ldots, s_{i}\right\}$, where $K_{i, j}{ }^{*} \subseteq \overline{V_{i} / N_{i}}$ is the unique field extension of $K_{i, j}$ with $\left[K_{i, j}{ }^{*}: K_{i, j}\right]=m$. Then $T^{*}=$ $\left\{T^{*}\left(V_{1}\right), \ldots, T^{*}\left(V_{n}\right)\right\}$ is a realizable $m^{2}$-consistent system for $\left\{V_{1}, \ldots, V_{n}\right\}$. 
Proof. If $m=1$, then $s_{i}=f_{i, j}=e_{i, j}=1$ for $i=1, \ldots, n$ and $j=1, \ldots, s_{i}$, by Definition 1.5, 2, so $S$ is realizable for $\left\{V_{1}, \ldots, V_{n}\right\}$, by Theorem 2.1(i), and $K_{i, j}=K_{i}$ for all $i, j$, so $F$ is a realization of $S=T^{*}$ for $\left\{V_{1}, \ldots, V_{n}\right\}$, so it may be assumed that $m>1$. If $s_{i}=1$ for some $i=1, \ldots, n$, then $S$ (resp., $T^{*}$ ) is a realizable $m$-consistent (resp., $m^{2}$-consistent) system for $\left\{V_{1}, \ldots, V_{n}\right\}$, by Theorem 2.1(i), so it may be assumed that $s_{i}>1$ for $i=1, \ldots, n$.

Since $n>1$, let $T_{1}\left(V_{n}\right)=\left\{\left(H_{n}, m, 1\right)\right\}$, where $H_{n} \subseteq \overline{V_{n} / N_{n}}$ is the unique field extension of $V_{n} / N_{n}$ with $\left[H_{n}:\left(V_{n} / N_{n}\right)\right]=m$. For $i=1, \ldots, n-1$ let $T_{1}\left(V_{i}\right)=S\left(V_{i}\right)$, and let $T_{1}=$ $\left\{T_{1}\left(V_{1}\right), \ldots, T_{1}\left(V_{n-1}\right), T_{1}\left(V_{n}\right)\right\}$, so $T_{1}$ is a realizable $m$-consistent system for $\left\{V_{1}, \ldots, V_{n}\right\}$, by Theorem 2.1(i). Let $L_{1}$ be a realization of $T_{1}$ for $\left\{V_{1}, \ldots, V_{n}\right\}$, so $L_{1}$ is a separable algebraic extension field of $F$ of degree $m$. For $i=1, \ldots, n$ let $\left(W_{i, j}, N_{i, j}\right)$ be the valuation rings of $L_{1}$ that lie over $V_{i}$. Then it follows from the prescription of $T_{1}$ that there are exactly $s_{i}$ such rings for $i=1, \ldots, n-1$, but only one such ring for $i=n$. Also, $W_{i, j} / N_{i, j}$ is $\left(V_{i} / N_{i}\right)$-isomorphic to $K_{i, j}$ and $N_{i} W_{i, j}=N_{i, j} e_{i, j}$ for $i=1, \ldots, n-1$ and $j=1, \ldots, s_{i}$, while $W_{n, 1} / N_{n, 1}$ is $\left(V_{n} / N_{n}\right)$-isomorphic to $H_{n}$ and $N_{n} W_{n, 1}=N_{n, 1}$.

For $T_{2}$ we use the fields $K_{i, j}{ }^{*}$ in the statement of this theorem, so $K_{i, j} \subseteq K_{i, j}{ }^{*}$ and $\left[K_{i, j}{ }^{*}\right.$ : $\left.K_{i, j}\right]=m$ for all $i, j$, so by Remark 2.6. 1 it follows, from $\left[H_{n}: K_{n}\right]=m$, that $H_{n} \subseteq K_{n, j}{ }^{*}$ and $\left[K_{n, j}{ }^{*}: H_{n}\right]=f_{n, j}$. With this in mind, let $T_{2}=\left\{T_{2}\left(W_{1,1}\right), \ldots, T_{2}\left(W_{n-1, s_{n-1}}\right), T_{2}\left(W_{n, 1}\right)\right\}$, where $T_{2}\left(W_{i, j}\right)=\left\{\left(K_{i, j}{ }^{*}, m, 1\right)\right\}$ for $i=1, \ldots, n-1$ and $j=1, \ldots, s_{i}$, and where $T_{2}\left(W_{n, 1}\right)$ $=\left\{\left(K_{n, j}{ }^{*}, f_{n, j}, e_{n, j}\right) \mid j=1, \ldots, s_{n}\right\}$. It is readily checked that $T_{2}$ is an $m$-consistent system for $\mathcal{W}:=\left\{W_{1,1}, \ldots, W_{n-1, s_{n-1}}, W_{n, 1}\right\}$. By Theorem 2.1(i) it is realizable for $\mathcal{W}$. Let $L$ be a realization of $T_{2}$ for $\mathcal{W}$, so $L$ is a separable algebraic extension field of $L_{1}$ of degree $m$ (so $L$ is a separable algebraic extension field of $F$ of degree $m^{2}$ ), and for $i=1, \ldots, n-1$ and $j=1, \ldots, s_{i}$ there exists a unique valuation $\operatorname{ring}\left(U_{i, j}, P_{i, j}\right)$ of $L$ that lies over $W_{i, j}$ (and then $U_{i, j} / P_{i, j}$ is $\left(W_{i, j} / N_{i, j}\right)$-isomorphic to $K_{i, j}{ }^{*}$ (and $W_{i, j} / N_{i, j} \supseteq\left(V_{i} / N_{i}\right)$, so $U_{i, j} / P_{i, j}$ is $\left(V_{i} / N_{i}\right)$-isomorphic to $\left.K_{i, j}{ }^{*}\right)$ and $N_{i, j} U_{i, j}=P_{i, j}$, so $\left.N_{i} U_{i, j}=N_{i, j}{ }^{e}{ }_{i, j}\right)$, while for $i=n$ there are exactly $s_{n}$ valuation rings $\left(U_{n, j}, P_{n, j}\right)$ that lie over $\left(W_{n, 1}, N_{n, 1}\right)$, and for $j=1, \ldots, s_{n}$, $U_{n, j} / P_{n, j}$ is $\left(W_{n, 1} / N_{n, 1}\right)$-isomorphic to $K_{n, j}{ }^{*}$ (and $W_{n, 1} / N_{n, 1} \supseteq\left(V_{n} / N_{n}\right)$, so $U_{n, j} / P_{n, j}$ is $\left(V_{n} / N_{n}\right)$-isomorphic to $\left.K_{n, j}{ }^{*}\right)$ and $N_{n, 1} U_{n, j}=P_{n, j}{ }^{e_{n, j}}$, so $N_{n} U_{n, j}=P_{n, j}{ }^{e_{n, j}}$. Also, since $U_{i, j} / P_{i, j}$ is a finite field for all $i, j$, it is a simple extension field of $V_{i} / N_{i}$ (concerning this, see Definition 1.5(1)). It therefore follows that $L$ is a realization of the $m^{2}$-consistent system $T^{*}$ 
$=\left\{T^{*}\left(V_{1}\right), \ldots, T^{*}\left(V_{n}\right)\right\}$ for $\left\{V_{1}, \ldots, V_{n}\right\}$, where $T^{*}\left(V_{i}\right)=\left\{\left(K_{i, j}{ }^{*}, m f_{i, j}, e_{i, j}\right) \mid j=1, \ldots, s_{i}\right\}$ for $i=1, \ldots, n$. Therefore $T^{*}$ is a realizable $m^{2}$-consistent system for $\left\{V_{1}, \ldots, V_{n}\right\}$.

Remark 2.8 The hypothesis in Theorem 2.7 that each $K_{i}=V_{i} / N_{i}$ is finite is often not essential. Specifically, if the set of extension fields of the $K_{i}$ have the following properties (a) - (c), then it follows from the proof of Theorem 2.7 that the conclusion holds, even though the $K_{i}$ are not finite: (a) For $i=1, \ldots, n$ and $j=1, \ldots s_{i}$ there exists a field $K_{i, j}{ }^{*}$ such that $\left[K_{i, j}{ }^{*}: K_{i, j}\right]=m$. (b) Each $K_{i, j}{ }^{*}$ is a simple extension of $K_{i}$. (c) There exists $i$ $\in\{1, \ldots, n\}$ (say $i=n$ ) such that there exists a simple extension field $H_{n}$ of $K_{n}$ of degree $m$ such that $H_{n} \subseteq K_{n, j}{ }^{*}$ for $j=1, \ldots, s_{n}$ (so $\left[K_{n, j}{ }^{*}: H_{n}\right]=f_{n, j}$ for $j=1, \ldots, s_{n}$ ).

Corollary 2.9 Let $R$ be a Noetherian domain, let $I$ be a nonzero proper ideal in $R$, let $\left(V_{1}, N_{1}\right), \ldots,\left(V_{n}, N_{n}\right)$ be the Rees valuation rings of $I$, let $m, s_{1}, \ldots, s_{n}$ be positive integers, and let $S=\left\{S\left(V_{1}\right), \ldots, S\left(V_{n}\right)\right\}$ be an arbitrary $m$-consistent system for $V_{1}, \ldots, V_{n}$, say $S\left(V_{i}\right)=\left\{\left(K_{i, j}, f_{i, j}, e_{i, j}\right) \mid j=1, \ldots, s_{i}\right\}$ for $i=1, \ldots, n$. Assume that each $V_{i} / N_{i}$ is finite. Then there exists a separable algebraic extension field $L$ of $R_{(0)}$ of degree $m^{2}$ such that, for each finite integral extension domain $A$ of $R$ with quotient field $L$ and for $i=1, \ldots, n, I A$ has exactly $s_{i}$ Rees valuation rings $\left(W_{i, j}, N_{i, j}\right)$ lying over $V_{i}$, and then, for $j=1, \ldots, s_{i}$, the Rees integer of $I A$ with respect to $W_{i, j}$ is $e_{i, j}$ and $\left[\left(W_{i, j} / N_{i, j}\right):\left(V_{i} / N_{i}\right)\right]=m f_{i, j}$.

Proof. As in the proof of Corollary 2.5, this follows immediately from Theorem 2.7

\section{Radical-power ideals.}

Let $D$ be an arbitrary Dedekind domain. A classical result states that each nonzero proper ideal $I$ of $D$ is a finite product of prime ideals. An application, Corollary 3.5, of the main result in this section, Theorem 3.3, shows that $I$ extends to a radical-power ideal in a suitable finite integral extension domain $E$ of $D$; in fact, we prove that $I E=(\operatorname{Rad}(I E))^{m}$, where $m=\left[E_{(0)}: D_{(0)}\right]$. To facilitate the statement and proof of the results in this section, we use the following notation and terminology. 
Notation 3.1 Let $D$ be a Dedekind domain with quotient field $F \neq D$, let $M_{1}, \ldots, M_{n}$ be distinct maximal ideals of $D$, and let $I=M_{1}{ }^{e_{1}} \cdots M_{n}{ }^{e_{n}}$ be an ideal in $D$, where $e_{1}, \ldots, e_{n}$ are positive integers. Then:

(3.1,1) For each finite integral extension domain $A$ of $D$ (including $D$ ) let $\mathbf{M}_{I}(A)=\{N \mid N$ is a maximal ideal in $A$ and $\left.N \cap D \in\left\{M_{1}, \ldots, M_{n}\right\}\right\}$.

$(\mathbf{3 . 1}, 2)$ Let $E$ be a finite integral extension Dedekind domain of $D$ and let $\mathbf{V}=\left\{E_{N} \mid N \in\right.$ $\left.\mathbf{M}_{I}(E)\right\}$. If $S$ is an $m$-consistent system for $\mathbf{V}$, then by abuse of terminology we sometimes say that $S$ is an $m$-consistent system for $\mathbf{M}_{I}(E)$, and when $N \in \mathbf{M}_{I}(E)$ we sometimes use $S(N)$ in place of $S\left(E_{N}\right)$.

Remark 3.2 With the notation of (3.1), let $S=\left\{S\left(M_{1}\right), \ldots, S\left(M_{n}\right)\right\}$ be a realizable $m$ consistent system for $\mathbf{M}_{I}(D)$, where $S\left(M_{i}\right)=\left\{\left(K_{i, j}, f_{i, j}, e_{i, j}\right) \mid j=1, \ldots, s_{i}\right\}$ for $i=$ $1, \ldots, n$. Let $L$ be a field that realizes $S$ for $\mathbf{M}_{I}(D)$ and let $E$ be the integral closure of $D$ in $L$. Then:

(3.2,1) $[L: F]=m$, and $L$ has distinct DVRs $\left(V_{i, 1}, N_{i, 1}\right), \ldots,\left(V_{i, s_{i}}, N_{i, s_{i}}\right)$ such that for each $i, j: V_{i, j} \cap F=D_{M_{i}} ; V_{i, j} / N_{i, j}$ is $D / M_{i}$-isomorphic to $\left.K_{i, j} ;\left[K_{i, j}: K_{i}\right)\right]=f_{i, j}$, where $K_{i}$ $=D / M_{i}$; and, $M_{i} V_{i, j}=N_{i, j}{ }^{e_{i, j}}$. Also, for $i=1, \ldots, n, V_{i, 1}, \ldots, V_{i, s_{i}}$ are all of the extensions of $D_{M_{i}}$ to $L$, so $\mathbf{M}_{I}(E)=\left\{N_{i, j} \cap E \mid i=1, \ldots, n\right.$ and $\left.j=1, \ldots, s_{i}\right\}$.

$(\mathbf{3 . 2}, 2) E$ is a Dedekind domain that is a finite separable integral extension domain of $D$, and $I E=M_{1}{ }^{e_{1}} \cdots M_{n}{ }^{e_{n}} E=P_{1,1} e_{1} e_{1,1} \cdots P_{n, s_{n}} e_{n} e_{n, s_{n}}$, where $P_{i, j}=N_{i, j} \cap E$ for $i=1, \ldots, n$ and $j=1, \ldots, s_{i}$.

Proof. (3.2.1) follows immediately from (a) - (c) of Definition 1.6.

For (3.2,2), $E$ is a Dedekind domain, by [19, Theorem 19, p. 281], and $E$ is a finite separable integral extension domain of $D$, by [19, Corollary 1, p. 265], since $L$ is a finite separable algebraic extension field of $F$. Also, $V_{i, j}=E_{P_{i, j}}$, so $I V_{i, j}=(I E) V_{i, j}=\left(I D_{M_{i}}\right) V_{i, j}$ $=\left(M_{i}{ }^{e_{i}} D_{M_{i}}\right) V_{i, j}=\left(M_{i} V_{i, j}\right)^{e_{i}}=N_{i, j}{ }_{i} e_{i, j}$. Since the ideals $P_{i, j}$ are the only prime ideals in $E$ that lie over $M_{i}$ (for $i=1, \ldots, n$ and $j=1, \ldots, s_{i}$ ) and since the $P_{i, j}$ are comaximal, it follows that $I E=P_{1,1} e_{1} e_{1,1} \ldots P_{n, s_{n}} e_{n} e_{n, s_{n}}$.

Theorem 3.3 is the main result of this section; it shows that every ideal $I$ as in Notation 3.1 extends to a radical-power ideal in some finite integral extension Dedekind domain. 
This theorem is proved in [10, (2.11.1)] by composing $n$ related consistent systems. We give this different proof here since it suggests the proof of the analogous "finite-residue-field degree" result given in Theorem 4.1 .

Theorem 3.3 With the notation of (3.1) and (3.2), assume that $n>1$. Then the system $S=\left\{S\left(M_{1}\right), \ldots, S\left(M_{n}\right)\right\}$ is a realizable $e_{1} \cdots e_{n}$-consistent system for $\mathbf{M}_{I}(D)$, where, for $i=1, \ldots, n, S\left(M_{i}\right)=\left\{\left(K_{i, j}, 1, \frac{e_{1} \cdots e_{n}}{e_{i}}\right) \mid j=1, \ldots, e_{i}\right\}$. Therefore there exists a Dedekind domain $E$ that is a finite separable integral extension domain of $D$ such that $[L: F]=$ $e_{1} \cdots e_{n}$, where $L$ (resp., $F$ ) is the quotient field of $E$ (resp., $D$ ), and, for $i=1, \ldots, n$, there exist exactly $e_{i}$ maximal ideals $N_{i, 1}, \ldots, N_{i, e_{i}}$ in $E$ that lie over $M_{i}$ and, for $j=1, \ldots, e_{i}$, $\left[\left(E / N_{i, j}\right):\left(D / M_{i}\right)\right]=1$ and $M_{i} E_{N_{i, j}}=N_{i, j}^{\frac{e_{1} \cdots e_{n}}{e_{i}}} E_{N_{i, j}}$, so $I E=(\operatorname{Rad}(I E))^{e_{1} \cdots e_{n}}$.

Proof. If $e_{h}=1$ for some $h=1, \ldots, n$, then since $K_{i, j} \cong D / M_{i}$ for all $i, j$, it follows that condition (1) of Definition 1.5] is satisfied, and it is readily checked that condition (2) of Definition 1.5 is satisfied with $m=e_{1} \cdots e_{n}$, so $S$ is an $e_{1} \cdots e_{n}$-consistent system for $\mathbf{M}_{I}(D)$. Since the cardinality of $S\left(M_{h}\right)$ is $e_{h}=1, S$ is realizable for $\mathbf{M}_{I}(D)$, by Theorem 2.1(i). Hence the system $S$ is a realizable $e_{1} \cdots e_{n}$-consistent system for $\mathbf{M}_{I}(D)$. Let $L$ be a realization of $S$ for $\mathbf{M}_{I}(D)$ (so $[L: F]=e_{1} \cdots e_{n}$, by (a) of Definition [1.6), and let $E$ be the integral closure of $D$ in $L$. Then $E$ is a Dedekind domain that is a finite separable integral extension domain of $D$, by Remark 3.2 .2 , and it readily follows from either Remark 3.2 .1 or the prescription given by $S$ that, for $i=1, \ldots, n$, there exist exactly $e_{i}$ maximal ideals $N_{i, 1}, \ldots, N_{i, e_{i}}$ in $E$ that lie over $M_{i}$ and, for $j=1, \ldots, e_{i}, E / N_{i, j} \cong D / M_{i}$ and $M_{i} E_{N_{i, j}}$ $=N_{i, j}{ }^{\frac{e_{1} \cdots e_{n}}{e_{i}}} E_{N_{i, j}}$, so $M_{i} E=\Pi_{j=1}^{e_{i}} N_{i, j} \frac{e_{1} \cdots e_{n}}{e_{i}}$. Therefore, since $I=M_{1}{ }^{e_{1}} \cdots M_{n}{ }^{e_{n}}$, it follows that $I E=\left(M_{1}^{e_{1}} \cdots M_{n}^{e_{n}}\right) E=\Pi_{i=1}^{n}\left[\left(\Pi_{j=1}^{e_{i}} N_{i, j} \frac{e_{1} \cdots e_{n}}{e_{i}}\right) e^{e_{i}}\right]$, so $I E=(\operatorname{Rad}(I E))^{e_{1} \cdots e_{n}}$. Thus it may be assumed that $e_{i}>1$ for $i=1, \ldots, n$.

Let $S_{1}=\left\{S_{1}\left(M_{1}\right), \ldots, S_{1}\left(M_{n}\right)\right\}$, where $S_{1}\left(M_{n}\right)=\left\{\left(\left(D / M_{n}\right), 1, e_{1} \cdots e_{n-1}\right)\right\}$ and for $i$ $=1, \ldots, n-1, S_{1}\left(M_{i}\right)=\left\{\left(K_{i, j}, 1, \frac{e_{1} \cdots e_{n-1}}{e_{i}}\right) \mid j=1, \ldots, e_{i}\right\}$. Then it follows as in the preceding paragraph that $S_{1}$ is a realizable $e_{1} \cdots e_{n-1}$-consistent system for $\mathbf{M}_{I}(D)$. Let $L_{1}$ be a realization of $S_{1}$ for $\mathbf{M}_{I}(D)$ (so $\left[L_{1}: F\right]=e_{1} \cdots e_{n-1}$, by (a) of Definition [1.6), and let $E_{1}$ be the integral closure of $D$ in $L_{1}$, so $E_{1}$ is a Dedekind domain that is a finite separable integral extension domain of $D$. Also, for $i=1, \ldots, n-1$ there exist exactly 
$e_{i}$ maximal ideals $Q_{i, 1}, \ldots, Q_{i, e_{i}}$ in $E_{1}$ that lie over $M_{i}$ and, for $j=1, \ldots, e_{i}, E_{1} / Q_{i, j} \cong$ $D / M_{i}$ and $M_{i}\left(E_{1}\right)_{Q_{i, j}}=Q_{i, j} \frac{{ }^{\frac{e_{1} \cdots e_{n-1}}{e_{i}}}}{e_{i}}\left(E_{1}\right)_{Q_{i, j}}$, so $M_{i} E_{1}=\Pi_{j=1}^{e_{i}} Q_{i, j} \frac{e_{1} \cdots e_{n-1}}{e_{i}}$. Further, there is a unique maximal ideal $Q_{n, 1}$ in $E_{1}$ that lies over $M_{n}, E_{1} / Q_{n, 1} \cong D / M_{n}$ and $M_{n}\left(E_{1}\right)_{Q_{n, 1}}$ $=Q_{n, 1}{ }^{e_{1} \cdots e_{n-1}}\left(E_{1}\right)_{Q_{n, 1}}$, so $M_{n} E_{1}=Q_{n, 1}{ }^{e_{1} \cdots e_{n-1}}$.

It follows that there are exactly $m^{\prime}=e_{1}+\cdots+e_{n-1}+1$ ideals $Q_{1,1}, \ldots, Q_{n, 1}$ in $\mathbf{M}_{I}\left(E_{1}\right)$, so let $S_{2}=\left\{S_{2}\left(Q_{1,1}\right), \ldots, S_{2}\left(Q_{1, e_{1}}\right), \ldots, S_{2}\left(Q_{n-1,1}\right) \ldots, S_{2}\left(Q_{n-1, e_{n-1}}\right), S_{2}\left(Q_{n, 1}\right)\right\}$, where $S_{2}\left(Q_{n, 1}\right)=\left\{\left(K_{n, j}, 1,1\right) \mid j=1, \ldots, e_{n}\right\}$, and for all other $(i, j), S_{2}\left(Q_{i, j}\right)=\left\{\left(\left(D / M_{i}\right), 1, e_{n}\right)\right\}$. Then it follows as in the second preceding paragraph that $S_{2}$ is a realizable $e_{n}$-consistent system for $\mathbf{M}_{I}\left(E_{1}\right)$. Let $L$ be a realization of $S_{2}$ for $\mathbf{M}_{I}\left(E_{1}\right)$ (so $\left[L: L_{1}\right]=e_{n}$, by (a) of Definition [1.6, so $[L: F]=e_{1} \cdots e_{n}$ ), and let $E$ be the integral closure of $E_{1}$ in $L$, so $E$ is a Dedekind domain that is a finite separable integral extension domain of $E_{1}$, so also of $D$. Also, for $i=1, \ldots, m^{\prime}-1$ and $j=1, \ldots, e_{i}$ there exists exactly one ideal $N_{i, j}$ in $\mathbf{M}_{I}(E)$ that lies over $Q_{i, j}, E / N_{i, j} \cong E_{1} / Q_{i, j}$, and $Q_{i, j} E_{N_{i, j}}=N_{i, j}{ }^{e_{n}} E_{N_{i, j}}$, so $Q_{i, j} E=N_{i, j}{ }^{e_{n}}$ (so it follows from the preceding paragraph that there exist exactly $e_{i}$ maximal ideals $N_{i, 1}, \ldots, N_{i, e_{i}}$ in $E$ that lie over $M_{i}$ and, for $i=1, \ldots, n-1$ and $j=1, \ldots, e_{i}, E / N_{i, j} \cong D / M_{i}$ and $M_{i} E_{N_{i, j}}=$ $N_{i, j} \frac{e_{1} \cdots e_{n}}{e_{i}} E_{N_{i, j}}$, so $\left.M_{i} E=\Pi_{j=1}^{e_{i}} N_{i, j} \frac{e_{1} \cdots e_{n}}{e_{i}}\right)$. And there exist exactly $e_{n}$ ideals $N_{n, 1}, \ldots, N_{n, e_{n}}$ in $\mathbf{M}_{I}(E)$ that lie over $Q_{n, 1}$ and, for $j=1, \ldots, e_{n}, E / N_{n, j} \cong E_{1} / Q_{n, 1}$ and $Q_{n, 1} E_{N_{n, j}}=$ $N_{n, j} E_{N_{n, j}}$, so $Q_{n, 1} E=\Pi_{j=1}^{e_{n}} N_{n, j}$ (so it follows from the preceding paragraph that there exist exactly $e_{n}$ maximal ideals $N_{n, 1}, \ldots, N_{n, e_{n}}$ in $E$ that lie over $M_{n}$ and, for $j=1, \ldots, e_{n}$, $E / N_{n, j} \cong D / M_{n}$ and $M_{n} E_{N_{n, j}}=N_{n, j} \frac{e_{1} \cdots e_{n}}{e_{n}} E_{N_{n, j}}$, so $\left.M_{n} E=\Pi_{j=1}^{e_{n}} N_{n, j} \frac{e_{1} \cdots e_{n}}{e_{n}}\right)$. It follows that $L$ is a realization of the $e_{1} \cdots e_{n}$-consistent system $S$ for $\mathbf{M}_{I}(D)$, (with $S$ as in the statement of this theorem), so $S$ is a realizable $e_{1} \cdots e_{n}$-consistent system for $\mathbf{M}_{I}(D)$.

Finally, $\left[\left(E / N_{i, j}\right):\left(D / M_{i}\right)\right]=1$ for $i=1, \ldots, n$ and $j=1, \ldots, e_{i}$, by the preceding paragraph, and, since $I=M_{1}^{e_{1}} \cdots M_{n}^{e_{n}}$ and $M_{i} E_{N_{i, j}}=N_{i, j}^{\frac{e_{1} \cdots e_{n}}{e_{i}}} E_{N_{i, j}}$ for $i=1, \ldots, n$, it follows that $I E=(\operatorname{Rad}(I E))^{e_{1} \cdots e_{n}}$.

Remark 3.4 (3.4.1) If no prime integer divides all of the $e_{i}$ in Theorem 3.3, we show in Theorem 3.11 that the exponent $e_{1} \cdots e_{n}$ in Theorem 3.3 can be replaced by the least common multiple of the $e_{i}$. So for example if $n=3$ and $\left(e_{1}, e_{2}, e_{3}\right)=(4,6,5)$, we get $I E=$ $(\operatorname{Rad}(I E))^{60}$ instead of $(\operatorname{Rad}(I E))^{120}$. See also Remark 3.12. 
(3.4,2) With the notation of Theorem 3.3, let $d$ be a common multiple of $e_{1}, \ldots, e_{n-1}$ and let $d^{*}=d e_{n}$. (Thus, for example, if $e_{1}=\cdots=e_{n-1}$, then $d^{*}=e_{1} e_{n}$ is (depending on $e_{1}$ and $n$ ) potentially considerably smaller than $e_{1} \cdots e_{n}$.) Then the following $d^{*}$-consistent system $S^{*}$ is realizable for $\mathbf{M}_{I}(D): S^{*}=\left\{S^{*}\left(M_{1}\right), \ldots, S^{*}\left(M_{n}\right)\right\}$ with $S^{*}\left(M_{i}\right)=\left\{\left(K_{i, j}, 1, \frac{d^{*}}{e_{i}}\right) \mid j=\right.$ $\left.1, \ldots, e_{i}\right\}$ for $i=1, \ldots, n$. Also, $I E^{*}=\left(\operatorname{Rad}\left(I E^{*}\right)\right)^{d^{*}}$, where $E^{*}$ is the integral closure of $D$ is a realization of $S^{*}$ for $\mathbf{M}_{I}(D)$.

Proof. For (3.4,2), the proof is the same as the proof of Theorem 3.3 by composing the following two realizable consistent systems $S_{1}{ }^{*}, S_{2}{ }^{*}$. Here, $S_{1}{ }^{*}=\left\{S_{1}{ }^{*}\left(M_{1}\right), \ldots, S_{1}{ }^{*}\left(M_{n}\right)\right\}$ with $S_{1}{ }^{*}\left(M_{i}\right)=\left\{\left(K_{i, j}, 1, \frac{d^{*}}{e_{i} e_{n}}\right) \mid j=1, \ldots, e_{i}\right\}$ for $i=1, \ldots, n-1$, and $S_{1}{ }^{*}\left(M_{n}\right)=$ $\left\{\left(\left(D / M_{n}\right), 1, \frac{d^{*}}{e_{n}}\right)\right\}$, so $S_{1}{ }^{*}$ is a realizable $\frac{d^{*}}{e_{n}}$-consistent system for $\mathbf{M}_{I}(D)$. Let $E_{1}{ }^{*}$ be the integral closure of $D$ is a realization $L_{1}{ }^{*}$ of $S_{1}{ }^{*}$ for $\mathbf{M}_{I}(D)$, and for $i=1, \ldots, n$ let $Q_{i, 1}, \ldots, Q_{i, e_{i}}$ be the maximal ideals in $E_{1}$ that lie over $M_{i}$. Let

$$
S_{2}{ }^{*}=\left\{S_{2}{ }^{*}\left(Q_{1,1}\right), \ldots, S_{2}{ }^{*}\left(Q_{n-1, e_{n-1}}\right), S_{2}{ }^{*}\left(Q_{n, 1}\right)\right\}
$$

with $S_{2}{ }^{*}\left(Q_{i, j}\right)=\left\{\left(\left(D / M_{i}\right), 1, e_{i}\right)\right\}$ for $i=1, \ldots, n-1$ and $j=1, \ldots, e_{i}$, and $S_{2}{ }^{*}\left(Q_{n, 1}\right)=$ $\left\{\left(K_{n, j}, 1,1\right) \mid j=1, \ldots, e_{n}\right\}$, so $S_{2}{ }^{*}$ is a realizable $e_{n}$-consistent system for $\mathbf{M}_{I}\left(E_{1}\right)$.

The following corollary is essentially given in [10, (2.10)], except for the exponent $e_{1} \cdots e_{n}$ that occurs here by using Theorem 3.3 .

Corollary 3.5 Let $I=M_{1}{ }^{e_{1}} \cap \cdots \cap M_{n}{ }^{e_{n}}$ be an irredundant primary decomposition of the nonzero proper ideal I of the Dedekind domain D. Then there exists a finite separable integral extension Dedekind domain $E$ of $D$ such that $I E=(\operatorname{Rad}(I E))^{m}$, where $m=$ $e_{1} \cdots e_{n}$

Proof. If $n=1$, then $I=M_{1}{ }^{e_{1}}=(\operatorname{Rad}(I))^{e_{1}}$, so the conclusion holds with $E=D$ and $m=e_{1}$. If $n>1$, then the conclusion follows immediately from Theorem 3.3 , since $I=$ $M_{1}{ }^{e_{1}} \cap \cdots \cap M_{n}^{e_{n}}=M_{1}^{e_{1}} \cdots M_{n}^{e_{n}}$.

Corollary 3.6 Let $k=\pi_{1}^{e_{1}} \cdots \pi_{n}^{e_{n}}$ be the factorization of the positive integer $k>1$ as a product of distinct prime integers $\pi_{i}$. . Then there exists an extension field $L$ of $\mathbb{Q}$ of degree $e_{1} \cdots e_{n}$ such that $k E=\left[\Pi_{i=1}^{n}\left(\Pi_{j=1}^{e_{i}} p_{i, j}\right)\right]^{e_{1} \cdots e_{n}}$, where $E$ is the integral closure of $\mathbb{Z}$ in $L$ and $\mathbf{M}_{k \mathbb{Z}}(E)=\left\{p_{1,1}, \ldots, p_{n, e_{n}}\right\}$. 
Remark 3.7 shows that $I$ sometimes extends to a radical power ideal in a simpler realizable consistent system.

Remark 3.7 With the notation of (3.1) and (3.2), assume 2 that, for $i=1, \ldots, n$, there exists a simple algebraic extension field $K_{i}{ }^{(1)}$ of $D / M_{i}$ such that $\left[K_{i}{ }^{(1)}:\left(D / M_{i}\right)\right]=e_{i}$. Then the system $S^{(1)}=\left\{S^{(1)}\left(M_{1}\right), \ldots, S^{(1)}\left(M_{n}\right)\right\}$, where $S^{(1)}\left(M_{i}\right)=\left\{\left(K_{i}^{(1)}, e_{i}, \frac{e_{1} \cdots e_{n}}{e_{i}}\right)\right\}$ for $i=$ $1, \ldots n$, is an $e_{1} \cdots e_{n}$-consistent system for $\mathbf{M}_{I}(D)$. By Theorem 2.1(i), it is realizable for $\mathbf{M}_{I}(D)$. Also, if $E$ is the integral closure of $D$ in a realization $L$ of $S^{(1)}$ for $\mathbf{M}_{I}(D)$, then $I E=J^{e_{1} \cdots e_{n}}$, where $J=\operatorname{Rad}(I E)$. More specifically, since $E$ is the integral closure of $D$ in a realization $L$ of $S^{(1)}$ for $\mathbf{M}_{I}(D)$, for $i=1, \ldots, n$, there exists a unique maximal ideal $N_{i}$ in $E$ that lies over $M_{i}$, and then $E / N_{i} \cong K_{i}^{(1)}$ and $M_{i} E_{N_{i}}=N_{i}^{\frac{e_{1} \cdots e_{n}}{e_{i}}} E_{N_{i}}$, so $M_{i} E=$ $N_{i}^{\frac{e_{1} \cdots e_{n}}{e_{i}}}$, so $I E=\left(\Pi_{i=1}^{n} M_{i}^{e_{i}}\right) E=\Pi_{i=1}^{n}\left(N_{i}^{\frac{e_{1} \cdots e_{n}}{e_{i}}}\right)^{e_{i}}=J^{e_{1} \cdots e_{n}}$, where $J=N_{1} \cdots N_{n}$.

Remark 3.8 Let $V_{i}=D_{M_{i}}$ and $S=\left\{S\left(V_{1}\right), \ldots, S\left(V_{n}\right)\right\}$ be an arbitrary $m$-consistent system for $\mathbf{M}_{I}(D)=\left\{M_{1}, \ldots, M_{n}\right\}$, where, for $i=1, \ldots, n, S\left(V_{i}\right)=\left\{\left(K_{i, j}, f_{i, j}, e_{i, j}\right) \mid j=\right.$ $\left.1, \ldots, s_{i}\right\}$. If we consider the $s_{i}, K_{i, j}$, and $f_{i, j}$ as fixed in the $m$-consistent system for $\mathbf{M}_{I}(D)$ and the $e_{i, j}$ as variables subject to the constraint $\sum_{j=1}^{s_{i}} e_{i, j} f_{i, j}=m$ for each $i$, then $S$ gives a map $\mathbb{N}_{+}{ }^{n} \rightarrow \mathbb{N}_{+}{ }^{t}$ (where $t=\sum_{i=1}^{n} s_{i}$ ) defined by

$$
\left(e_{1}, \ldots, e_{n}\right) \mapsto\left(e_{1} e_{1,1}, \ldots, e_{1} e_{1, s_{1}}, \ldots, e_{n} e_{n, 1}, \ldots, e_{n} e_{n, s_{n}}\right) .
$$

If we are only interested in the projective equivalence class of $I E$, it seems appropriate to consider the induced map given by $S: \mathbb{N}_{+}{ }^{n} \rightarrow \mathbf{P}^{t}\left(\mathbb{N}_{+}\right)=\mathbb{N}_{+}{ }^{t} / \sim$, where $\left(a_{1}, \ldots, a_{t}\right) \sim$ $\left(b_{1}, \ldots, b_{t}\right)$ if $\left(a_{1}, \ldots, a_{t}\right)=\left(c b_{1}, \ldots, c b_{t}\right)$ for some $c \in \mathbb{Q}$. In this case, Theorem 2.3 shows that the equations $\sum_{j=1}^{s_{i}} e_{i, j} f_{i, j}=m$ are the only restrictions on the image of this map into $\mathbf{P}^{t}\left(\mathbb{N}_{+}\right)$. From this point of view, if we want an equation $I E=(\operatorname{Rad}(I E))^{k}$ for some finite separable integral extension Dedekind domain $E$ of $D$ and for some positive integer $k$, then it is not necessary to compose two realizable consistent systems, as in the proof of Theorem 3.3. Indeed, it suffices to observe that we have an $m$-consistent system $S=$ $\left\{S\left(M_{1}\right), \ldots, S\left(M_{n}\right)\right\}$, where $m=e_{1} \cdots e_{n}$ and $S\left(M_{i}\right)=\left\{\left(K_{i, j}, 1, \frac{e_{1} \cdots e_{n}}{e_{i}}\right) \mid j=1, \ldots, e_{i}\right\}$ for $i=1, \ldots, n$ (realizable or not), and then apply Theorem 2.3 .

\footnotetext{
${ }^{2} D$ may have a residue field $D / M_{i}$ that has no extension field $K_{i}{ }^{(1)}$ with $\left[K_{i}{ }^{(1)}:\left(D / M_{i}\right)\right]=e_{i}$; for example, $D / M_{i}$ may be algebraically closed, see also Example 3 in 15 .
} 
To extend Theorem 3.3 to ideals in Noetherian domains of altitude one, we use the following result from [10].

Proposition 3.9 [10, (2.6)] Let $R$ be a Noetherian domain of altitude one with quotient field $F$, let $I$ be a nonzero proper ideal in $R$, let $L$ be a finite algebraic extension field of $F$, let $E$ be the integral closure of $R$ in $L$, and assume there exist distinct maximal ideals $N_{1}, \ldots, N_{n}$ of $E$ and positive integers $k_{1}, \ldots, k_{n}, h$ such that $I E=\left(N_{1} k_{1} \cdots N_{n}{ }^{k_{n}}\right)^{h}$. Then there exists a finite integral extension domain $A$ of $R$ with quotient field $L$ and distinct maximal ideals $P_{1}, \ldots, P_{n}$ of $A$ such that, for $i=1, \ldots, n$ :

(i) $P_{i} E=N_{i}$.

(ii) $E / N_{i} \cong A / P_{i}$.

(iii) $(I A)_{a}=\left(\left(P_{1}^{k_{1}} \cdots P_{n}^{k_{n}}\right)^{h}\right)_{a}$.

The following corollary is the same as [10, (2.8.2)], except for the explicit exponent $e_{1} \cdots e_{n}$ given here.

Corollary 3.10 Let $R$ be a Noetherian domain of altitude one, let $I$ be a nonzero proper ideal in $R$, let $R^{\prime}$ be the integral closure of $R$ in its quotient field, and let $I R^{\prime}=M_{1}{ }^{e_{1}} \cdots M_{n}{ }^{{ }_{n}}$ be a normal primary decomposition of $I R^{\prime}$. Then there exists a finite separable integral extension domain $A$ of $R$ such that $(I A)_{a}=\left((\operatorname{Rad}(I A))^{e_{1} \cdots e_{n}}\right)_{a}$, and if $A^{\prime}$ denotes the integral closure of $A$ in its quotient field, then for each $P \in \mathbf{M}_{I}(A)$ we have: (i) $P A^{\prime}$ is a maximal ideal, and (ii) $A^{\prime} / P A^{\prime} \cong A / P$.

Proof. If $n=1$, then $I R^{\prime}=\left(\operatorname{Rad}\left(I R^{\prime}\right)\right)^{e_{1}}$ and $R^{\prime}$ is a Dedekind domain, so the conclusion follows from Proposition 3.9.

If $n>1$, then by hypothesis there are exactly $n$ distinct maximal ideals $M_{1}, \ldots, M_{n}$ in $R^{\prime}$ that contain $I R^{\prime}$ and $I R^{\prime}=M_{1}^{e_{1}} \cdots M_{n}{ }^{e_{n}}$. Also, $R^{\prime}$ is a Dedekind domain, so by Theorem 3.3 there exists a finite separable integral extension Dedekind domain $E$ of $R^{\prime}$ such that $I E=(\operatorname{Rad}(I E))^{e_{1} \cdots e_{n}}$. Then $E$ is the integral closure of $R$ in the quotient field of $E$; the conclusions follow from this, together with Proposition 3.9. 
An additional way to compose realizable consistent systems to obtain a Dedekind domain $E$ as in Theorem 3.3 is discussed in [10, (2.11.2)]. We consider [10, (2.11.2)] again here in Proposition 3.11 because we want to add an observation on the exponent $e_{1} \cdots e_{n}$. It gives a different inductive way to prove Theorem 3.3 when the exponents $e_{1}, \ldots, e_{n}$ have no common integer prime divisors and replaces the exponent and degree $e_{1} \cdots e_{n}$ in Theorem 3.3 with a smaller exponent and degree $d$. This also gives corresponding different versions of Corollaries 3.6 and 3.10. In case the exponents $e_{1}, \ldots, e_{n}$ do have common integer prime divisors, see Remark 3.12 .

Proposition 3.11 With the notation of (3.1) and (3.2), assume that $n>1$ and that no prime integer divides each $e_{i}$. Let $d=p_{1}{ }^{m_{1}} \cdots p_{k}{ }^{m_{k}}$ be the least common multiple of $e_{1}, \ldots, e_{n}$, where $p_{1}, \ldots, p_{k}$ are distinct prime integers and $m_{1}, \ldots, m_{k}$ are positive integers. Then the system $\mathbf{S}=\left\{\mathbf{S}\left(M_{1}\right), \ldots, \mathbf{S}\left(M_{1}\right)\right\}$ for $\mathbf{M}_{I}(D)$, where, for $i=1, \ldots, n, \mathbf{S}\left(M_{i}\right)$ $=\left\{\left(K_{i, j}, 1, \frac{d}{e_{i}}\right) \mid j=1, \ldots, e_{i}\right\}$, is a realizable d-consistent system for $\mathbf{M}_{I}(D)$. Also, if $E$ is the integral closure of $D$ in a realization $L$ of $\mathbf{S}$ for $\mathbf{M}_{I}(D)$, then $I E=(\operatorname{Rad}(I E))^{d}$.

Proof. The proof is similar to the proof of [10, (2.11.1)]. There exists a chain of rings

$$
(*) \quad D=E_{(0)} \subset E_{1} \subset \cdots \subset E_{k}=E,
$$

where each $E_{h}(h=1, \ldots, k)$ is the integral closure of $E_{h-1}$ in a realization $L_{h}$ of a realizable $p_{h}{ }^{m_{h}}$-consistent system $\mathbf{S}_{h}$ for $\mathbf{M}_{I}\left(E_{h-1}\right)$. To describe the consistent systems used to obtain these rings $E_{h}$ we first need the factorizations of each $e_{i}$. So, for $i=1, \ldots, n$ let $e_{i}=p_{1}{ }^{c_{i, 1}} \cdots p_{k}{ }^{c_{i, k}}$, so $0 \leq c_{i, j} \leq m_{j}$ for $j=1, \ldots, k$, since $d=p_{1}{ }^{m_{1}} \cdots p_{k}{ }^{m_{k}}$. With this notation, it will now be shown that, for $h=1, \ldots, k, E_{h}$ has, for $i=1, \ldots, n$, exactly $t_{h, i}=p_{1}{ }^{c_{i, 1}} \cdots p_{h}{ }^{c_{i, h}}$ maximal ideals $P_{i, 1}, \ldots, P_{i, t_{h, i}}$ that lie over $M_{i}$ and, for $j=1, \ldots, t_{h, i}$, $E_{h} / P_{i, j} \cong D / M_{i}$ and $M_{i}\left(E_{h}\right)_{P_{i, j}}=P_{i, j}{ }^{r_{h, i}}\left(E_{h}\right)_{P_{i, j}}$, where $r_{h, i}=p_{1}{ }^{m_{1}-c_{i, 1}} \cdots p_{h}{ }^{m_{h}-c_{i, h}}$.

For the first step, let $e_{i}=p_{1}{ }^{c_{i, 1}} d_{i, 1}$ with $p_{1} \Lambda d_{i, 1}$, so $0 \leq c_{i, 1} \leq m_{1}$ for each $i$. It may be assumed that $c_{1,1} \geq c_{2,1} \geq \cdots \geq c_{n, 1}$ (so $c_{1,1}=m_{1}$ and $c_{n, 1}=0$ (by the hypothesis that no prime divides all $\left.e_{i}\right)$ ), and let $\mathbf{S}_{1}=\left\{\mathbf{S}_{1}\left(M_{1}\right), \ldots, \mathbf{S}_{1}\left(M_{n}\right)\right\}$, where $\mathbf{S}_{1}\left(M_{i}\right)=$ $\left\{\left(K_{i, j}, 1, p_{1}{ }^{m_{1}-c_{i, 1}}\right) \mid j=1, \ldots, s_{i}=p_{1}{ }^{c_{i, 1}}\right\}$ for $i=1, \ldots, n$. Then $\mathbf{S}_{1}$ is a $p_{1}{ }^{m_{1}}$-consistent system for $\mathbf{M}_{I}(D)$, and since $c_{n, 1}=0$, it is realizable for $\mathbf{M}_{I}(D)$. Let $E_{1}$ be the integral clo- 
sure of $D$ in a realization $L_{1}$ of $\mathbf{S}_{1}$ for $\mathbf{M}_{I}(D)$. Then by Remark $3.2,2, I E_{1}=\prod_{i=1}^{n}\left(M_{i}{ }^{e_{i}} E_{1}\right)$

$=$

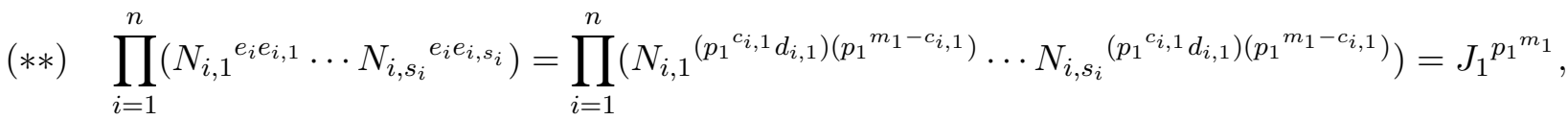

where $J_{1}=\prod_{i=1}^{n}\left(N_{i, 1} d_{i, 1} \cdots N_{i, s_{i}} d_{i, 1}\right)$, and $\prod_{i=1}^{n} d_{i, 1}{ }^{s_{i}}=\prod_{i=1}^{n} d_{i, 1}{ }^{p_{1}{ }^{c_{i, 1}}}$ has $p_{2}, \ldots, p_{k}$ as its prime integer factors.

Assume that $h>1$ and that $E_{h-1}$ has been constructed to have the properties in the second preceding paragraph, so, in particular, for $i=1, \ldots, n, \mathbf{M}_{I}\left(E_{h-1}\right)$ has exactly $t_{h-1, i}$ maximal ideals $P_{i, 1}, \ldots, P_{i, t_{h-1, i}}$ that lie over $M_{i}$ and, for $j=1, \ldots, t_{h-1, i}, E_{h-1} / P_{i, j} \cong$ $D / M_{i}$ and $M_{i}\left(E_{h-1}\right)_{P_{i, j}}=P_{i, j}{ }^{r_{h-1, i}}\left(E_{h-1}\right)_{P_{i, j}}$.

To get $E_{h}$ from $E_{h-1}$, let $\mathbf{S}_{h}=\left\{\mathbf{S}_{h}\left(P_{1,1}\right), \ldots, \mathbf{S}_{h}\left(P_{n, t_{h-1, n}}\right)\right\}$, where

$$
\mathbf{S}_{h}\left(P_{i, j}\right)=\left\{\left(K_{i, j, l}, 1, p_{h}{ }^{m_{h}-c_{i, h}}\right) \mid l=1, \ldots, p_{h}{ }^{c_{i, h}}\right\} \text { for all } i, j .
$$

Then it is readily checked that $\mathbf{S}_{h}$ is a $p_{h}{ }^{m_{h}}$-consistent system for $\mathbf{M}_{I}\left(E_{h-1}\right)$, and it is realizable for $\mathbf{M}_{I}\left(E_{h-1}\right)$, by Theorem[2.1(i). It then follows from the prescription of $\mathbf{S}_{h}$ that the integral closure $E_{h}$ of $E_{h-1}$ in a realization $L_{h}$ of $\mathbf{S}_{h}$ for $\mathbf{M}_{I}\left(E_{h-1}\right)$ has the properties of $E_{h}$ in the third preceding paragraph.

It therefore follows that $[L: F]=p_{1}{ }^{m_{1}} \cdots p_{k}{ }^{m_{k}}=d$, where $L$ (resp. $F$ ) is the quotient field of $E=E_{k}$ (resp., $D=E_{(0)}$ ) and that $E$ is a realization of the system $\mathbf{S}$ for $\mathbf{M}_{I}(D)$ (with $\mathbf{S}$ as in the statement of this theorem), so $\mathbf{S}$ is a realizable $d$-consistent system for $\mathbf{M}_{I}(D)$. Finally, it follows from (**), applied in each of the $k$ steps, that $I E=(\operatorname{Rad}(I E))^{d}$.

Remark 3.12 Concerning the hypothesis in Proposition 3.11 that no prime integer divides all $e_{i}$, if, on the contrary, $\pi$ is a prime integer that divides each $e_{i}$, then let $c$ be the greatest common divisor of $e_{1}, \ldots, e_{n}$. For $i=1, \ldots, n$ define $k_{i}$ by $e_{i}=c k_{i}$, and let $I_{0}$ $=M_{1}{ }^{k_{1}} \cdots M_{n}{ }^{k_{n}}$, so $I_{0}{ }^{c}=\left(\prod_{i=1}^{n} M_{i}^{k_{i}}\right)^{c}=\prod_{i=1}^{n} M_{i}^{e_{i}}=I$ and no prime integer divides all $k_{i}$. Therefore, if the ring $E$ of Theorem 3.3 is constructed for $I_{0}$ in place of $I$, then $I_{0} E=$ $\left(\operatorname{Rad}\left(I_{0} E\right)\right)^{d}$, where $d$ is the least common multiple of $k_{1}, \ldots, k_{n}$, so $I E=(\operatorname{Rad}(I E))^{d c}$.

Theorem 3.3 shows that there exist finite separable integral extension domains $E$ of $D$ such that $I E$ is a radical-power ideal. Proposition 3.13 characterizes the conditions a 
realizable $m$-consistent system $S^{\prime}$ for $\mathbf{M}_{I}(D)$ must satisfy in order that $I E=J^{t}$ for some radical ideal $J$ in $E$ and for some positive integer $t$.

Proposition 3.13 Let $D$ be a Dedekind domain with quotient field $F \neq D$, let $M_{1}, \ldots, M_{n}$ $(n>1)$ be distinct maximal ideals of $D$, let $I=M_{1}{ }^{e_{1}} \cdots M_{n}{ }^{e_{n}}$ be an ideal in $D$, where $e_{1}, \ldots, e_{n}$ are positive integers, and let $m$ be a positive integer. Let $S^{\prime}=\left\{S^{\prime}\left(M_{1}\right), \ldots, S^{\prime}\left(M_{n}\right)\right\}$ be a realizable m-consistent system for $\left\{D_{M_{1}}, \ldots, D_{M_{n}}\right\}$, where $S^{\prime}\left(M_{i}\right)=\left\{\left(K_{i, j}, f_{i, j}, e_{i, j}\right) \mid\right.$ $\left.j=1, \ldots, s_{i}\right\}$ for $i=1, \ldots, n$, and let $E$ be the integral closure of $D$ in a finite separable field extension $L$ of $F$ which realizes $S^{\prime}$ for $\left\{D_{M_{1}}, \ldots, D_{M_{n}}\right\}$, so $[L: F]=m$. Then the following hold:

(3.13,1) $I E=J^{t}$ for some radical ideal $J$ in $E$ and for some positive integer $t$ if and only if the products $e_{i} e_{i, j}$ are equal for all $i, j$, and then $J=\operatorname{Rad}(I E)$ and $e_{i} e_{i, j}=t$.

(3.13,2) If IE $=J^{m}$ (as in Theorem 3.3 and Proposition 3.11), then $\sum_{j=1}^{s_{i}} f_{i, j}=e_{i}$ for $i$ $=1, \ldots, n$.

(3.13,3) If IE $=J^{t}$, as in (3.13,1), and if no prime integer divides each $e_{i}$, then $m$ is a positive multiple of $t$ and $t$ (and hence $m$ ) is a positive multiple of each $e_{i}$.

Proof. For (3.13,1), it is clear that if $I E=J^{t}$ for some radical ideal $J$ in $E$, then $J=$ $\operatorname{Rad}(I E)$. Therefore let $J=\operatorname{Rad}(I E)=P_{1} \cdots P_{k}$, for distinct prime ideals $P_{1}, \ldots, P_{k}$ of $E$. Then by Remark 3.2, $I E=\prod_{i=1}^{n}\left(P_{i, 1} e_{i} e_{i, 1} \cdots P_{i, s_{i}}{ }{ }_{i} e_{i, s_{i}}\right)$. Thus by uniqueness of primary decompositions in a Dedekind domain, it follows that $J^{t}=I E$ if and only if $t=e_{i} e_{i, j}$ for each $i$ and $j$, hence (3.13,1) holds.

For (3.13,2), by (2) in the definition of a consistent system we have $m=\sum_{j=1}^{s_{i}} e_{i, j} f_{i, j}$ for $i=1, \ldots, n$. Therefore if $(3.13,1)$ holds and if $t=m(=[L: F])$, then $m=t=e_{i} e_{i, j}$ for all $i, j$, so $e_{i, j}=\frac{m}{e_{i}}$ for $i=1, \ldots, n$ and $j=1, \ldots, s_{i}$. Substituting $\frac{m}{e_{i}}$ for $e_{i, j}$ and multiplying by $e_{i}$ we get $m e_{i}=m \sum_{j=1}^{s_{i}} f_{i, j}$ for each $i$, so the conclusion follows by cancelling $m$.

For $(3.13,3)$, if $(3.13,1)$ holds, then as in the proof of $(3.13,2)$ we have $e_{i, j}=\frac{t}{e_{i}}$ for all $i, j$ and $m=\sum_{j=1}^{s_{i}} e_{i, j} f_{i, j}$ for all $i$. Substituting $\frac{t}{e_{i}}$ for $e_{i, j}$ and multiplying by $e_{i}$ we get $m e_{i}$ $=t \sum_{j=1}^{s_{i}} f_{i, j}$ for each $i$. Since no prime divides each $e_{i}$, we get $m=t m^{\prime}$ for some $m^{\prime} \in \mathbb{N}_{+}$. Therefore, since $t=e_{i} e_{i, j}$ for all $i, j, t$ and $m$ are positive multiples of each $e_{i}$. 


\section{Finite-residue-field degree analogs.}

Under the assumption that each of the residue fields $D / M_{i}$ is finite, the results in this section are "finite-residue-field degree" analogs of the results in Section 3. Theorem 4.1 is the main result in this section; it is a finite-residue-field degree analog of Theorem 3.3 .

Theorem 4.1 With the notation of (3.1) and (3.2), assume that $n>1$ and that each $K_{i}=$ $D / M_{i}$ is finite. For $i=1, \ldots, n$ let $f_{i}$ be a positive integer such that $\left[K_{i}: F_{i}\right]=f_{i}$ for some subfield $F_{i}$ of $K_{i}$, and let $K_{i}{ }^{\prime} \subseteq \overline{K_{i}}$ be the unique extension field of $K_{i}$ of degree $\frac{f_{1} \cdots f_{n}}{f_{i}}$, where $\overline{K_{i}}$ is a fixed algebraic closure of $K_{i}$. Then the system $T=\left\{T\left(M_{1}\right), \ldots, T\left(M_{n}\right)\right\}$ is a realizable $m$-consistent system for $\mathbf{M}_{I}(D)$, where $m=f_{1} \cdots f_{n}$ and $T\left(M_{i}\right)=\left\{\left(K_{i, j}, \frac{f_{1} \cdots f_{n}}{f_{i}}, 1\right) \mid\right.$ $\left.j=1, \ldots, f_{i}\right\}$ for $i=1, \ldots, n$ (with $K_{i, j}=K_{i}^{\prime}$ for $j=1, \ldots, f_{i}$ ). Therefore there exists a Dedekind domain $E$ that is a finite separable integral extension domain of $D$ such that $[L: F]=m$ (where $L$ (resp., F) is the quotient field of $E$ (resp., D)) and, for $i=$ $1, \ldots, n$, there exist exactly $f_{i}$ maximal ideals $N_{i, 1}, \ldots, N_{i, f_{i}}$ in $E$ that lie over $M_{i}$ and, for $j=1, \ldots, f_{i}, M_{i} E_{N_{i, j}}=N_{i, j} E_{N_{i, j}}$ and $\left[\left(E / N_{i, j}\right): K_{i}\right]=\frac{f_{1} \cdots f_{n}}{f_{i}}$, so $\left[\left(E / N_{i, j}\right): F_{i}\right]=m$.

Proof. The proof is similar to the proof of Theorem 3.3. Specifically, if $f_{h}=1$ for some $h=1, \ldots, n$, then $T\left(M_{h}\right)$ has $s_{h}=f_{h}=1$, so the system $T$ is a realizable $f_{1} \cdots f_{n^{-}}$ consistent system for $\mathbf{M}_{I}(D)$, by Theorem 2.1(i), and the integral closure $E$ of $D$ in a realization $L$ of $T$ for $\mathbf{M}_{I}(D)$ has the desired properties, so it may be assumed that $f_{i}$ $>1$ for all $i$. Then the desired ring $E$ is obtained by composing the following two systems $T_{1}$ (to get the Dedekind domain $E_{1}$ from $D$ ) and $T_{2}$ (to get the Dedekind domain $E$ from $\left.E_{1}\right)$. Here, $T_{1}=\left\{T_{1}\left(M_{1}\right), \ldots, T_{1}\left(M_{n}\right)\right\}$, where $T_{1}\left(M_{n}\right)=\left\{\left(K_{n, 1}, f_{1} \cdots f_{n-1}, 1\right)\right\}$ and for $i=1, \ldots, n-1, T_{1}\left(M_{i}\right)=\left\{\left(H_{i, j}, \frac{f_{1} \cdots f_{n-1}}{f_{i}}, 1\right) \mid j=1, \ldots, f_{i}\right\}$ (with $K_{i} \subseteq H_{i, j}$ $\subseteq K_{i, j}$; such $H_{i, j}$ exist, by Remark 2.6.1 , so $\left[K_{i, j}: H_{i, j}\right]=f_{n}$ ). It follows from Theorem 2.1(i) that $T_{1}$ is a realizable $f_{1} \cdots f_{n-1}$-consistent system for $\mathbf{M}_{I}(D)$ and that there are exactly $m^{\prime}=f_{1}+\cdots+f_{n-1}+1$ ideals $Q_{1,1}, \ldots, Q_{n-1, f_{n-1}}, Q_{n, 1}$ in $\mathbf{M}_{I}\left(E_{1}\right)$, where $E_{1}$ is the integral closure of $D$ in a realization $L_{1}$ of $T_{1}$ for $\mathbf{M}_{I}(D)$. Therefore let $T_{2}$ $=\left\{T_{2}\left(Q_{1,1}\right), \ldots, T_{2}\left(Q_{1, f_{1}}\right), \ldots, T_{2}\left(Q_{n-1,1}\right), \ldots, T_{2}\left(Q_{n-1, f_{n-1}}\right), T_{2}\left(Q_{n, 1}\right)\right\}$, where $T_{2}\left(Q_{n, 1}\right)=$ $\left\{\left(K_{n, j}, 1,1\right) \mid j=1, \ldots, f_{n}\right\}$, and for all other $(i, j), T_{2}\left(Q_{i, j}\right)=\left\{\left(K_{i, j}, f_{n}, 1\right)\right\}$. (Note that, by hypothesis, $E_{1} / Q_{n, 1} \cong K_{n, 1}=\cdots=K_{n, f_{n}}$.) It follows that $T_{2}$ is a $f_{n}$-consistent system 
for $\mathbf{M}_{I}\left(E_{1}\right)$, and it is realizable for $\mathbf{M}_{I}\left(E_{1}\right)$, by Theorem 2.1(i). Let $E$ be the integral closure of $E_{1}$ in a realization of $T_{2}$ for $\mathbf{M}_{I}\left(E_{1}\right)$. Then the $E / N_{n, j}$ are $E_{1} / Q_{n, 1}$-isomorphic to $K_{n, j}$ and $E_{1} / Q_{n, 1} \supseteq K_{n}$, so the $E / N_{n, j}$ are $K_{n}$-isomorphic to $K_{n, j}=K_{n, 1}=K_{n}{ }^{\prime}$. Also, by construction, for $i=1, \ldots, n-1$ and $j=1, \ldots, f_{i}, E / N_{i, j}$ is $E_{1} / Q_{i, j}$-isomorphic to $K_{i, j}$ and $E / Q_{i, j} \supseteq K_{i}$, so $E / N_{i, j}$ is $K_{i}$-isomorphic to $K_{i, j}$. Further, the $K_{i, j}$ are finite and contain $K_{i}$, so they are simple extensions of $K_{i}$. Therefore it follows as in the third paragraph of the proof of Theorem 3.3 that a realization $L$ of $T_{2}$ for $\mathbf{M}_{I}\left(E_{1}\right)$ is, in fact, a realization of $T$ for $\mathbf{M}_{I}(D)$ (with $T$ as in the statement of this theorem), so $T$ is a a realizable $f_{1} \cdots f_{n}$-consistent system for $\mathbf{M}_{I}(D)$.

Finally, it follows from the prescription given by $T$ that, for $i=1, \ldots, n$ and $j=1, \ldots, f_{i}$, $M_{i} E_{N_{i, j}}=N_{i, j} E_{N_{i, j}}$ and $\left[\left(E / N_{i, j}\right): K_{i}\right]=\frac{f_{1} \cdots f_{n}}{f_{i}}$, so $\left[\left(E / N_{i, j}\right): F_{i}\right]=f_{1} \cdots f_{n}$.

Remark 4.2 (4.2.1) The hypothesis in Theorem 4.1 that each $K_{i}=D_{i} / M_{i}$ is finite is often not essential. Specifically, if the set of extension fields of the $K_{i}$ have the following properties (a) - (c), then it follows from the proof of Theorem 4.1 that the conclusion holds, even though the $K_{i}$ are not finite: (a) For $i=1, \ldots, n, K_{i}$ has a subfield $F_{i}$ such that $\left[K_{i}: F_{i}\right]=f_{i}$. (b) With $m=f_{1} \cdots f_{n}$, for $i=1, \ldots, n K_{i}$ has (not necessarily distinct) simple extension fields $K_{i, 1}, \ldots, K_{i, f_{i}}$ such that $\left[K_{i, j}: K_{i}\right]=\frac{m}{f_{i}}$. (c) For $i=1, \ldots, n-1, K_{i}$ has simple extension fields $H_{i, j}$ such that $\left[H_{i, j}: K_{i}\right]=\frac{f_{1} \cdots f_{n-1}}{f_{i}}$ and such that $H_{i, j} \subseteq K_{i, j}$ (so $\left.\left[K_{i, j}: H_{i, j}\right]=f_{n}\right)$.

(4.2,2) With the notation of Theorem 4.1, let $d$ be a common multiple of $f_{1}, \ldots, f_{n-1}$ and let $d^{*}=d f_{n}$. (Thus, for example, if $f_{1}=\cdots=f_{n-1}$, then $d^{*}=f_{1} f_{n}$ is (depending on $f_{1}$ and $n$ ) potentially considerably smaller than $f_{1} \cdots f_{n}$.) Then the following $d^{*}$-consistent system $T^{*}$ is realizable for $\mathbf{M}_{I}(D): T^{*}=\left\{T^{*}\left(M_{1}\right), \ldots, T^{*}\left(M_{n}\right)\right\}$ with $T^{*}\left(M_{i}\right)=\left\{\left(K_{i, j}, \frac{d^{*}}{f_{i}}, 1\right) \mid j=\right.$ $\left.1, \ldots, f_{i}\right\}$ for $i=1, \ldots, n$ (with $K_{i, j}=K_{i}{ }^{*}$ for $j=1, \ldots, f_{i}$, where $K_{i}{ }^{*} \subseteq \overline{K_{i}}$ is the unique extension field of $K_{i}$ of degree $\left.d^{*}\right)$. Also, $\left[E^{*} / N_{i, j}: F_{i}\right]=d^{*}$ for all $i, j$, where $E^{*}$ is the integral closure of $D$ is a realization of $T^{*}$ for $\mathbf{M}_{I}(D)$.

Proof. For $(4.2,2)$, the proof is the same as the proof of Theorem 3.3 by composing the following two realizable consistent systems $T_{1}{ }^{*}, T_{2}{ }^{*}$. Here, $T_{1}{ }^{*}=\left\{T_{1}{ }^{*}\left(M_{1}\right), \ldots, T_{1}{ }^{*}\left(M_{n}\right)\right\}$ with $T_{1}{ }^{*}\left(M_{i}\right)=\left\{\left(H_{i, j}, \frac{d^{*}}{f_{i} f_{n}}, 1\right) \mid j=1, \ldots, f_{i}\right\}$ for $i=1, \ldots, n-1$ (with $K_{i} \subseteq H_{i, j} \subseteq K_{i, j}$, 
so $\left.\left[K_{i, j}: H_{i, j}\right]=f_{n}\right)$, and $T_{1}^{*}\left(M_{n}\right)=\left\{\left(K_{n, 1}, \frac{d^{*}}{f_{n}}, 1\right)\right\}$, so $T_{1}^{*}$ is a realizable $\frac{d^{*}}{f_{n}}$-consistent system for $\mathbf{M}_{I}(D)$. Let $E_{1}{ }^{*}$ be the integral closure of $D$ is a realization $L_{1}{ }^{*}$ of $T_{1}{ }^{*}$ for $\mathbf{M}_{I}(D)$, and for $i=1, \ldots, n$ let $Q_{i, 1}, \ldots, Q_{i, f_{i}}$ be the maximal ideals in $E_{1}$ that lie over $M_{i}$. Let $T_{2}{ }^{*}=\left\{T_{2}{ }^{*}\left(Q_{1,1}\right), \ldots, T_{2}{ }^{*}\left(Q_{n-1, f_{n-1}}\right), T_{2}{ }^{*}\left(Q_{n, 1}\right)\right\}$ with $T_{2}{ }^{*}\left(Q_{i, j}\right)=\left\{\left(K_{i, j}, f_{n}, 1\right)\right\}$ for $i$ $=1, \ldots, n-1$ and $j=1, \ldots, f_{i}$, and $T_{2}{ }^{*}\left(Q_{n, 1}\right)=\left\{\left(K_{n, j}, 1,1\right) \mid j=1, \ldots, f_{n}\right\}$, so $T_{2}{ }^{*}$ is a realizable $f_{n}$-consistent system for $\mathbf{M}_{I}\left(E_{1}\right)$.

Corollary 4.3 is a special case of Theorem 4.1 it is a finite-residue-field degree analog of Corollary 3.6

Corollary 4.3 Let $D$ be the ring of integers of an algebraic number field $F$ and let $M_{1}, \ldots, M_{n}$ $(n>1)$ be distinct maximal ideals in $D$. For $i=1, \ldots, n$ let $\mathbb{Z} / \pi_{i} \mathbb{Z}$ be the prime subfield of $D / M_{i}$ (possibly $\pi_{i}=\pi_{j}$ for some $\left.i \neq j \in\{1, \ldots, n\}\right)$ and let $f_{i}=\left[\left(D / M_{i}\right):\left(\mathbb{Z} / \pi_{i} \mathbb{Z}\right)\right]$. Then there exists a Dedekind domain $E$ that is a finite (separable) integral extension domain of $D$ such that, for $i=1, \ldots, n$, there exist exactly $f_{i}$ maximal ideals $p_{i, j}$ in $E$ that lie over $M_{i}$, and then, for $j=1, \ldots, f_{i}, M_{i} E_{p_{i, j}}=p_{i, j} E_{p_{i, j}}$ and $\left[\left(E / p_{i, j}\right):\left(\mathbb{Z} / \pi_{i} \mathbb{Z}\right)\right]=f_{1} \cdots f_{n}$.

Proof. This follows immediately from Theorem 4.1 .

Remark 4.4 corresponds to Remark 3.8 .

Remark 4.4 Let $D$ be a Dedekind domain with quotient field $F \neq D$, let $M_{1}, \ldots, M_{n}$ be distinct maximal ideals of $D$, and assume that $D / M_{i}$ is finite for $i=1, \ldots, n$. For $i=$ $1, \ldots, n$ let $f_{i}$ be a positive integer, and assume there exists a subfield $F_{i}$ of $D / M_{i}$ such that $\left[\left(D / M_{i}\right): F_{i}\right]=f_{i}$. Let $T=\left\{T\left(V_{1}\right), \ldots, T\left(V_{n}\right)\right\}$ be an arbitrary $m$-consistent system for $\mathbf{M}_{I}(D)=\left\{M_{1}, \ldots, M_{n}\right\}$, where, for $i=1, \ldots, n, T\left(V_{i}\right)=\left\{\left(K_{i, j}, f_{i, j}, e_{i, j}\right) \mid j=1, \ldots, s_{i}\right\}$. If we consider the $s_{i}, K_{i, j}$, and $e_{i, j}$ as fixed in the $m$-consistent system for $\mathbf{M}_{I}(D)$ and the $f_{i, j}$ as variables subject to the constraint $\sum_{j=1}^{s_{i}} e_{i, j} f_{i, j}=m$ for each $i$, then $T$ gives a map $\mathbb{N}_{+}{ }^{n} \rightarrow \mathbb{N}_{+}{ }^{t}$ (where $t=\sum_{i=1}^{n} s_{i}$ ) defined by

$$
\left(f_{1}, \ldots, f_{n}\right) \mapsto\left(f_{1} f_{1,1}, \ldots, f_{1} f_{1, s_{1}}, \ldots, f_{n} f_{n, 1}, \ldots, f_{n} f_{n, s_{n}}\right)
$$

and Theorem 2.7 shows that the equations $\sum_{j=1}^{s_{i}} e_{i, j} f_{i, j}=m$ are the only restrictions on the image of the induced map $S: \mathbb{N}_{+}{ }^{n} \rightarrow \mathbf{P}^{t}\left(\mathbb{N}_{+}\right)=\mathbb{N}_{+}{ }^{t} / \sim$, where $\left(a_{1}, \ldots, a_{t}\right) \sim\left(b_{1}, \ldots, b_{t}\right)$ if 
$\left(a_{1}, \ldots, a_{t}\right)=\left(c b_{1}, \ldots, c b_{t}\right)$ for some $c \in \mathbb{Q}$. From this point of view, if we want an equation $\left[\left(E / Q_{i, j}\right): F_{i}\right]=k$ for all $i, j$, for some finite separable integral extension Dedekind domain $E$ of $D$ and for some positive integer $k$, then it is not necessary to compose two realizable consistent systems, as in the proof of Theorem 4.1. Indeed, it suffices to observe that we have an $m$-consistent system $T=\left\{T\left(M_{1}\right), \ldots, T\left(M_{n}\right)\right\}$, where $m=f_{1} \cdots f_{n}$ and $T\left(M_{i}\right)=$ $\left\{\left(K_{i, j}, \frac{f_{1} \cdots f_{n}}{f_{i}}, 1\right) \mid j=1, \ldots, f_{i}\right\}$ for $i=1, \ldots, n$ (realizable or not), and then apply Theorem 2.7

Corollary 4.5 is a finite-residue-field degree analog of Corollary 3.10. Since hypotheses on infinite residue fields can sometimes be replaced by the hypotheses that the residue fields have cardinality greater than or equal to a given positive integer, Corollary 4.5 may be useful in this regard.

Corollary 4.5 Let $R$ be a Noetherian domain of altitude one, let I be a nonzero proper ideal in $R$, let $R^{\prime}$ be the integral closure of $R$ in its quotient field, let $I R^{\prime}=M_{1}{ }^{e_{1}} \cdots M_{n}{ }^{e_{n}}(n>1)$ be a normal primary decomposition of $I R^{\prime}$, and for $i=1, \ldots, n$ let $\left[\left(R^{\prime} / M_{i}\right):\left(R /\left(M_{i} \cap R\right)\right)\right]$ $=g_{i}$. For $i=1, \ldots, n$ assume that $R^{\prime} / M_{i}$ is finite, let $f_{i}$ be a positive integer, and assume that $\left[\left(R /\left(M_{i} \cap R\right)\right): F_{i}\right]=f_{i}$, where $F_{i}$ is a subfield of $R /\left(M_{i} \cap R\right)$. Then there exists a finite separable integral extension domain $A$ of $R$ such that, for all $P \in \mathbf{M}_{I}(A),\left[(A / P): F_{i}\right]=$ $\Pi_{i=1}^{n} f_{i} g_{i}=\left[A_{(0)}: R_{(0)}\right]$. Also, $A$ may be chosen so that, with $A^{\prime}$ the integral closure of $A$ in $A_{(0)}$, there exist exactly $f_{i} g_{i}$ maximal ideals $P_{i, j}$ in $A$ such that $P_{i, j} A^{\prime} \cap R^{\prime}=M_{i}$ and, for all $P \in \mathbf{M}_{I}(A)$ it holds that $P A^{\prime} \in \mathbf{M}_{I}\left(A^{\prime}\right)$ and $A / P \cong A^{\prime} /\left(P A^{\prime}\right)$.

Proof. Since $R^{\prime}$ is a Dedekind domain and $\left[\left(R^{\prime} / M_{i}\right): F_{i}\right]=f_{i} g_{i}$ for $i=1, \ldots, n$, it follows from Theorem 4.1 that there exists a Dedekind domain $E$ that is a finite separable integral extension domain of $R^{\prime}$ such that $\left[A_{(0)}: R_{(0)}\right]=\Pi_{i=1}^{n} f_{i} g_{i}$ and, for $i=1, \ldots, n$, there exist exactly $f_{i} g_{i}$ maximal ideals $N_{i, 1}, \ldots, N_{i, f_{i} g_{i}}$ in $E$ that lie over $M_{i}$ and, for $j=1, \ldots, f_{i} g_{i}$, $M_{i} E_{N_{i, j}}=N_{i, j} E_{N_{i, j}}$ and $\left[\left(E / N_{i, j}\right):\left(R^{\prime} / M_{i}\right)\right]=\frac{f_{1} g_{1} \cdots f_{n} g_{n}}{f_{i} g_{i}}$, so $\left[\left(E / N_{i, j}\right): F_{i}\right]=\Pi_{i=1}^{n} f_{i} g_{i}$. The conclusions follow from this, together with Proposition 3.9.

Part of Theorem 4.1 shows that if each residue field $D / M_{i}$ is finite and $F_{i}$ is a subfield of $D / M_{i}$ such that $\left[\left(D / M_{i}\right): F_{i}\right]=f_{i}$, then there exists a finite separable integral extension 
domain $E$ of $D$ such that $\left[E_{(0)}: D_{(0)}\right]=\left[\left(E / N_{i, j}\right): F_{i}\right]=f_{1} \cdots f_{n}$ for all $i, j$ (=m, say). Proposition 4.6 characterizes the conditions a realizable $m$-consistent system $T^{\prime}$ for $\mathbf{M}_{I}(D)$ must satisfy in order that $\left[\left(E / N_{i, j}\right): F_{i}\right]=f_{1} \cdots f_{n}$ for all $i, j$.

Proposition 4.6 Let $D$ be a Dedekind domain with quotient field $F \neq D$, let $M_{1}, \ldots, M_{n}$ $(n>1)$ be distinct maximal ideals of $D$, and assume that $K_{i}=D / M_{i}$ is finite for $i=$ $1, \ldots, n$. For $i=1, \ldots, n$ let $f_{i}$ be a positive integer such that $\left[K_{i}: F_{i}\right]=f_{i}$ for some subfield $F_{i}$ of $K_{i}$. Let $m$ be a positive integer and let $T^{\prime}=\left\{T^{\prime}\left(M_{1}\right), \ldots, T^{\prime}\left(M_{n}\right)\right\}$ be a realizable $m$-consistent system for $\mathbf{M}_{I}(D)$, where, for $i=1, \ldots, n, T^{\prime}\left(M_{i}\right)=\left\{\left(K_{i, j}, f_{i, j}, e_{i, j}\right) \mid j=\right.$ $\left.1, \ldots, s_{i}\right\}$, and let $E$ be the integral closure of $D$ in a realization $L$ of $T^{\prime}$ for $\mathbf{M}_{I}(D)$, so $[L: F]=m$. Then the following hold:

(4.6,1) There exists a positive integer $t$ such that $\left[\left(E / N_{i, j}\right): F_{i}\right]=t$ for all $i, j$ if and only if the products $f_{i} f_{i, j}$ are equal for all $i, j$, and then $t=f_{i} f_{i, j}$.

(4.6.2) If $\left[\left(E / N_{i, j}\right): F_{i}\right]=m$ for all $i, j$ (as in Theorem 4.1), then $\sum_{j=1}^{s_{i}} e_{i, j}=f_{i}$ for $i=$ $1, \ldots, n$.

(4.6.3) If $\left[\left(E / N_{i, j}\right): F_{i}\right]=t$ for all $i, j$, as in 4.6.1), and if no prime integer divides each $f_{i}$, then $m$ is a positive multiple of $t$ and $t$ (and hence $m$ ) is a positive multiple of each $f_{i}$.

Proof. For [4.61), by hypothesis $\left[\left(E / N_{i, j}\right): K_{i}\right]=f_{i, j}$ and $\left[K_{i}: F_{i}\right]=f_{i}$ for all $i, j$, so it follows that $\left[\left(E / N_{i, j}\right): F_{i}\right]=t$ for all $i, j$ if and only if $f_{i} f_{i, j}=t$ for all $i, j$, hence (4.6.1) holds.

For (4.6,2), by (2) in the definition of a consistent system we have $m=\sum_{j=1}^{s_{i}} e_{i, j} f_{i, j}$ for $i=1, \ldots, n$. Therefore if (4.6.1) holds and if $t=m(=[L: F])$, then $m=t=f_{i} f_{i, j}$ for all $i, j$, so $f_{i, j}=\frac{m}{f_{i}}$ for $i=1, \ldots, n$ and $j=1, \ldots, s_{i}$. Substituting $\frac{m}{f_{i}}$ for $f_{i, j}$ and multiplying by $f_{i}$ we get $m f_{i}=m \sum_{j=1}^{s_{i}} e_{i, j}$ for each $i$, so the conclusion follows by cancelling $m$.

For (4.6,3), if (4.6.1) holds, then as in the proof of 44.6,2) we have $f_{i, j}=\frac{t}{f_{i}}$ for all $i, j$ and $m=\sum_{j=1}^{s_{i}} e_{i, j} f_{i, j}$ for all $i$. Substituting $\frac{t}{f_{i}}$ for $f_{i, j}$ and multiplying by $f_{i}$ we get $m f_{i}$ $=t \sum_{j=1}^{s_{i}} e_{i, j}$ for each $i$. Since no prime integer divides each $f_{i}$, we get $m=t m^{\prime}$ for some $m^{\prime} \in \mathbb{N}_{+}$. Therefore, since $t=f_{i} f_{i, j}$ for all $i, j, t$ and $m$ are positive multiples of each $f_{i}$. 


\section{Finite residue fields and radical-power ideals.}

Theorem 5.1 is the main result in this section; it combines the main theorems of the preceding two sections.

Theorem 5.1 With the notation of (3.1) and (3.2) (so $I=M_{1}{ }^{e_{1}} \cdots M_{n}{ }^{e_{n}}$, where $n>$ 1 and the $e_{i}$ are positive integers), assume that each $K_{i}=D / M_{i}$ is finite and let $\overline{K_{i}}$ be a fixed algebraic closure of $K_{i}$. For $i=1, \ldots, n$ let $f_{i}$ be a positive integer such that $K_{i}$ is an extension field of a subfield $F_{i}$ with $\left[K_{i}: F_{i}\right]=f_{i}$, and let $K_{i}{ }^{*}$ be the unique extension field of $K_{i}$ of degree $e_{1} \cdots e_{n} f_{1} \cdots f_{n}$ that is contained in $\overline{K_{i}}$. Then the system $U=\left\{U\left(M_{1}\right), \ldots, U\left(M_{n}\right)\right\}$ is a realizable $e_{1} \cdots e_{n} f_{1} \cdots f_{n}$-consistent system for $\mathbf{M}_{I}(D)$,

where, for $i=1, \ldots, n, U\left(M_{i}\right)=\left\{\left(K_{i, j}, \frac{f_{1} \cdots f_{n}}{f_{i}}, \frac{e_{1} \cdots e_{n}}{e_{i}} \mid j=1, \ldots, e_{i} f_{i}\right\}\right.$ (with $K_{i, j}=K_{i}{ }^{*}$ for $\left.j=1, \ldots, e_{i} f_{i}\right)$. Therefore there exists a separable algebraic extension field $L$ of degree $e_{1} \cdots e_{n} f_{1} \cdots f_{n}$ over the quotient field $F$ of $D$, and a finite integral extension Dedekind domain $E$ of $D$ with quotient field $L$ such that, for $i=1, \ldots, n$, there are exactly $e_{i} f_{i}$ maximal ideals $N_{i, 1}, \ldots, N_{i, e_{i} f_{i}}$ in $E$ that lie over $M_{i}$, and it holds that $\left[\left(E / N_{i, j}\right): F_{i}\right]=$ $f_{1} \cdots f_{n}$ for all $i$ and $j$, and $I E=(\operatorname{Rad}(I E))^{e_{1} \cdots e_{n}}=\left(N_{1,1} \cdots N_{e_{n} f_{n}}\right)^{e_{1} \cdots e_{n}}$.

Proof. Let $S^{*}=\left\{S^{*}\left(M_{1}\right), \ldots, S^{*}\left(M_{n}\right)\right\}$, where $S^{*}\left(M_{i}\right)=\left\{\left(G_{i, j}, 1, \frac{e_{1} \cdots e_{n}}{e_{i}}\right) \mid j=1, \ldots e_{i}\right\}$ for $i=1, \ldots n$ (with $G_{i, j}=K_{i}$ for all $i, j$ ). Then $S^{*}$ is a realizable $e_{1} \cdots e_{n}$-consistemt system for $\mathbf{M}_{I}(D)$, by Theorem [3.3. Let $L_{1}$ be a realization of $S^{*}$ for $\mathbf{M}_{I}(D)$ (so $L_{1}$ is a separable algebraic extension field of $F$ of degree $e_{1} \cdots e_{n}$ ), and let $E_{1}$ be the integral closure of $D$ in $L_{1}$. Thus by Theorem 3.3, for $i=1, \ldots, n$ there exist exactly $e_{i}$ maximal ideals $Q_{i, 1}, \ldots, Q_{i, e_{i}}$ in $E_{1}$ that lie over $M_{i}, I E_{1}=\left(Q_{1,1} \cdots Q_{n, e_{n}}\right)^{e_{1} \cdots e_{n}}$, and $E_{1} / Q_{i, j}$ is $K_{i}$-isomorphic to $K_{i}$.

Let $T^{*}=\left\{T^{*}\left(Q_{1,1}\right), \ldots, T^{*}\left(Q_{n, e_{n}}\right)\right\}$, where $T^{*}\left(Q_{i, j}\right)=\left\{\left(H_{i, j, k}, \frac{f_{1} \cdots f_{n}}{f_{i}}, 1\right) \mid k=1, \ldots f_{i}\right\}$ for all $i, j$ (where $H_{i, j, k}$ is one of the $e_{i} f_{i}$ ideals $K_{i, j}$ in the set $U\left(M_{i}\right)$. Then $T^{*}$ is a realizable $f_{1} \cdots f_{n}$-consistemt system for $\mathbf{M}_{I}\left(E_{1}\right)$, by Theorem 4.1. Let $L$ be a realization of $T^{*}$ for $\mathbf{M}_{I}\left(E_{1}\right)$ (so $L$ is a separable algebraic extension field of $L_{1}$ of degree $f_{1} \cdots f_{n}$, so $L$ is a separable algebraic extension field of $F$ of degree $e_{1} \cdots e_{n} f_{1} \cdots f_{n}$ ), and let $E$ be the integral closure of $E_{1}$ in $L$. Thus by Theorem 4.1, for $i=1, \ldots, n$ and $j=1, \ldots e_{n}$ 
there exist exactly $f_{i}$ maximal ideals $N_{i, j, 1}, \ldots, N_{i, j, f_{i}}$ in $E$ that lie over $Q_{i, j}$ (so be resubscripting there are exactly $e_{i} f_{i}$ maximal ideals $N_{i, 1}, \ldots, N_{i, e_{i} f_{i}}$ in $E$ that lie over $\left.M_{i}\right), I E_{1}$ $=\left(N_{1,1} \cdots N_{n, e_{n} f_{n}}\right)^{e_{1} \cdots e_{n}} *$, and it is readily checked that $E / N_{i, j}$ is $K_{i}$-isomorphic to $K_{i, j}$. Therefore $E$ is a Dedekind domain that has exactly $e_{i} f_{i}$ maximal ideals that lie over $M_{i}$ (for $i=1, \ldots, n$ ) and that have the ramification and residue field extension properties that are specified by $U$ (with $U$ as in the statement of this theorem). Therefore $L$ is a realization of $U$ for $\mathbf{M}_{I}(D)$, so $U$ is a realizable $e_{1} \cdots e_{n} f_{1} \cdots f_{n}$-consistent system for $\mathbf{M}_{I}(D)$ and $E$ is the integral closure of $D$ in $L$ and has the properties prescribed by $U$.

Remark 5.2 It is readily seen that, alternately, Theorem 5.1 could be proved by first applying Theorem 4.1 to $D$ to obtain a finite integral extension Dedekind domain $E_{2}$ of $D$ with the desired residue field extension properties and no ramification of any $M_{1}, \ldots, M_{n}$, and then apply Theorem 3.3 to $E_{2}$ to yield the desired Dedekind domain, say $E^{\prime}$.

Proposition 5.3 is related to Theorem 5.1 , but does not follow immediately from it. It does not require the residue fields $D / M_{i}$ to be finite, but it does require they have a finite extension of a specific degree.

Proposition 5.3 With the notation of (3.1) and (3.2), assume that $n>1$ and that $D / M_{i}$ has a simple algebraic extension field $H_{i}$ of degree $e_{1} \cdots e_{n}$ for $i=1, \ldots, n$. Then the $\left(e_{1} \cdots e_{n}\right)^{2}$-consistent system $S^{*}=\left\{S^{*}\left(M_{1}\right), \ldots, S^{*}\left(M_{n}\right)\right\}$ for $\mathbf{M}_{I}(D)$ is realizable for $\mathbf{M}_{I}(D)$, where $S^{*}\left(M_{i}\right)=\left\{\left(K_{i, j}, e_{1} \cdots e_{n}, \frac{e_{1} \cdots e_{n}}{e_{i}}\right) \mid j=1, \ldots, e_{i}\right\}$ for $i=1, \ldots, n$ (where $K_{i, j}$ is $\left(D / M_{i}\right)$-isomorphic to $\left.H_{i}\right)$. Therefore there exists a separable algebraic extension field $L$ of the quotient field $F$ of $D$ of degree $\left(e_{1} \cdots e_{n}\right)^{2}$ and a finite separable integral extension Dedekind domain $E$ of $D$ with quotient field $L$ such that, for $i=1, \ldots, n$, there are exactly $e_{i}$ maximal ideals $N_{i, 1}, \ldots, N_{i, e_{i}}$ in $E$ that lie over $M_{i},\left[\left(E / N_{i, j}\right):\left(D / M_{i}\right)\right]=e_{1} \cdots e_{n}$ for all $i$ and $j$, and $I E=(\operatorname{Rad}(I E))^{e_{1} \cdots e_{n}}$.

Proof. Let $T=\left\{T\left(M_{1}\right), \ldots T\left(M_{n}\right)\right\}$, where $T\left(M_{i}\right)=\left\{\left(H_{i}, e_{1} \cdots e_{n}, 1\right)\right\}$ for $i=1, \ldots, n$. Then $T$ is realizable $e_{1} \cdots e_{n}$-consistent system for $\mathbf{M}_{I}(D)$, by Theorem 2.1(i), so the integral closure $E_{1}$ of $D$ in a realization $L_{1}$ of $T$ for $\mathbf{M}_{I}(D)$ has a unique maximal ideal $N_{i}$ that lies over $M_{i}$ for $\left.i=1, \ldots, n\right)$ and then $E_{1} / N_{i}$ is $D / M_{i}$-isormophic to $H_{i}$ and $M_{i} E_{1}=$ 
$N_{i}$. Let $S=\left\{S\left(N_{1}\right), \ldots, S\left(N_{n}\right)\right\}$, where $S\left(N_{i}\right)=\left\{\left(K_{i, j}, 1, \frac{e_{1} \cdots e_{n}}{e_{i}}\right) \mid j=1, \ldots, e_{i}\right\}$ for $i=$ $1, \ldots, n$ (so the $K_{i, j}$ are $\left(A / M_{i}\right)$-isomorphic to $H_{i}$ for $\left.j=1, \ldots, e_{i}\right)$. Then $S$ is a realizable $e_{1} \cdots e_{n}$-consistent system for $\mathbf{M}_{I}\left(E_{1}\right)$, by Theorem 3.3 applied to $\mathbf{M}_{I}\left(E_{1}\right)$. Therefore it is readily checked that the integral closure $E$ of $E_{1}$ in a realization $L$ of $S$ for $\mathbf{M}_{I}\left(E_{1}\right)$ has the properties prescribed by $S^{*}$ for $\mathbf{M}_{I}(D)$ (with $S^{*}$ as in the statement of this theorem). Therefore $L$ is a realization of $S^{*}$ for $\mathbf{M}_{I}(D)$, so $S^{*}$ is realizable for $\mathbf{M}_{I}(D)$.

If Proposition 5.3 is applied to $D=\mathbb{Z}$ and $I=72 \mathbb{Z}$, for example, then it follows that there exists a field $L$ of degree 36 over $\mathbb{Q}$ such that the integral closure $E$ of $\mathbb{Z}$ in $L$ has exactly three maximal ideals $p_{1,1}, p_{1,2}, p_{1,3}$ that lie over $2 \mathbb{Z}$ and exactly two maximal ideals $p_{2,1}, p_{2,2}$ that lie over $3 \mathbb{Z}, 72 E=\left(p_{1,1} p_{1,2} p_{1,3} p_{2,1} p_{2,2}\right)^{6},\left[\left(E / p_{1, j}\right):(\mathbb{Z} / 2 \mathbb{Z})\right]=6$ for $j=1,2,3$, and $\left[\left(E / p_{2, j}\right):(\mathbb{Z} / 3 \mathbb{Z})\right]=6$ for $j=1,2$.

Corollary 5.4 Let $R$ be a Noetherian domain of altitude one, let $I$ be a nonzero proper ideal in $R$, let $R^{\prime}$ be the integral closure of $R$ in its quotient field, let $I R^{\prime}=M_{1}{ }^{e_{1}} \cdots M_{n}{ }^{e_{n}}$ $(n>1)$ be a normal primary decomposition of $I R^{\prime}$.

(5.4.1) Assume that $R^{\prime} / M_{i}$ is finite for $i=1, \ldots, n$, let $\left[\left(R^{\prime} / M_{i}\right):\left(R /\left(M_{i} \cap R\right)\right)\right]=g_{i}$, and let $f_{i}$ be a positive integer such that $\left[\left(R /\left(M_{i} \cap R\right)\right): F_{i}\right]=f_{i}$ for some subfield $F_{i}$ of $R /\left(M_{i} \cap R\right)$. Then there exists a finite separable integral extension domain $A$ of $R$ such that $\left[A_{(0)}: R_{(0)}\right]=\Pi_{i=1}^{n} e_{i} f_{i} g_{i}$ and, for $i=1, \ldots, n$, there exist exactly $e_{i} f_{i} g_{i}$ maximal ideals $P_{i, j}$ $\in \mathbf{M}_{I}(A)$ such that, for $j=1, \ldots, e_{i} f_{i} g_{i}: P_{i, j} A^{\prime} \in \mathbf{M}_{I}\left(A^{\prime}\right) ; P_{i, j} A^{\prime} \cap R^{\prime}=M_{i} ;\left[\left(A / P_{i, j}\right): F_{i}\right]$ $=\Pi_{i=1}^{n} f_{i} g_{i} ;$ and, $(I A)_{a}=\left(\left[\Pi_{i=1}^{n}\left(\Pi_{j=1}^{e_{i} f_{i} g_{i}} P_{i, j}\right)\right]^{e_{1} \cdots e_{n}}\right)_{a}$.

(5.4,2) Assume that, for $i=1, \ldots, n, R^{\prime} / M_{i}$ has a simple algebraic extension field of degree $e_{1} \cdots e_{n}$. Then there exists a finite separable integral extension domain $A$ of $R$ such that $\left[A_{(0)}: R_{(0)}\right]=\left(\Pi_{i=1}^{n} e_{i}\right)^{2}$ and, for $i=1, \ldots, n$, there exist exactly $e_{i}$ maximal ideals $P_{i, j} \in$ $\mathbf{M}_{I}(A)$ such that, for $j=1, \ldots, e_{i}: P_{i, j} A^{\prime} \in \mathbf{M}_{I}\left(A^{\prime}\right) ; P_{i, j} A^{\prime} \cap R^{\prime}=M_{i} ;\left[\left(A / P_{i, j}\right):\left(R^{\prime} / M_{i}\right)\right]$ $=\Pi_{i=1}^{n} e_{i} ;$ and, $(I A)_{a}=\left(\left[\Pi_{i=1}^{n}\left(\Pi_{j=1}^{e_{i}} P_{i, j}\right)\right]^{e_{1} \cdots e_{n}}\right)_{a}$.

Proof. For (5.4.1), since $R^{\prime}$ is a Dedekind domain, it follows from Theorem 5.1 that there exists a finite separable integral extension Dedekind domain $E$ of $R$ such that $\left[E_{(0)}\right.$ : $\left.R_{(0)}\right]=\Pi_{i=1}^{n} e_{i} f_{i} g_{i}$ and, for $i=1, \ldots, n$, there exist exactly $e_{i} f_{i} g_{i}$ maximal ideals $N_{i, j} \in$ $\mathbf{M}_{I}(E)$ such that, for $j=1, \ldots, e_{i} f_{i} g_{i}: N_{i, j} \cap R^{\prime}=M_{i} ;\left[\left(E / P_{i, j}\right): F_{i}\right]=\Pi_{i=1}^{n} f_{i} g_{i} ;$ and, 
$I E=\left[\Pi_{i=1}^{n}\left(\Pi_{j=1}^{e_{i} f_{i} g_{i}} N_{i, j}\right)\right]^{e_{1} \cdots e_{n}}$. Therefore the conclusions follow from this, together with Proposition 3.9 .

The proof of $(5.4,2)$ is similar, but use Proposition 5.3 in place of Theorem 5.1.

The final result in this section follows immediately from combining Propositions 3.13 and 4.6 .

Remark 5.5 With the notation of (3.1) and (3.2) (so $I=M_{1}{ }^{e_{1}} \cdots M_{n}{ }^{e_{n}}$ ) assume that each $K_{i}=D / M_{i}$ is finite with a subfield $F_{i}$ such that $\left[K_{i}: F_{i}\right]=f_{i}$. Let $S=\left\{S\left(M_{1}\right), \ldots, S\left(M_{n}\right)\right\}$ with $S\left(M_{i}\right)=\left\{\left(K_{i, j}, f_{i, j}, e_{i, j}\right) \mid j=1, \ldots, s_{i}\right\}$ for $i=1, \ldots, n$ be a realizable $m$-consistent system for $\mathbf{M}_{I}(D)$ and let $E$ be the integral closure of $D$ in a realization $L$ of $S$ for $\mathbf{M}_{I}(D)$. Then there exist positive integers $t_{1}$ and $t_{2}$ such that $I E=(\operatorname{Rad}(I E))^{t_{1}}$ and such that, for $i=1, \ldots, n,\left[(E / N): F_{i}\right]=t_{2}$ for all maximal ideals $N$ in $E$ that lie over $M_{i}$ if and only if $t_{1}=e_{i} e_{i, j}$ and $t_{2}=f_{i} f_{i, j}$ for all $i, j$, and then $e_{i}=\sum_{i=1}^{s_{i}} f_{i, j}$ and $f_{i}=\sum_{i=1}^{s_{i}} e_{i, j}$.

\section{References}

[1] C. Ciupercă, W. J. Heinzer, L. J. Ratliff, Jr., and D. E. Rush, Projectively equivalent ideals and Rees valuations, J. Algebra 282 (2004), 140-156.

[2] C. Ciupercă, W. J. Heinzer, L. J. Ratliff, Jr., and D. E. Rush, Projectively full ideals in Noetherian rings, J. Algebra 304 (2006), 73-93.

[3] C. Ciupercă, W. J. Heinzer, L. J. Ratliff, Jr., and D. E. Rush, Projectively full ideals in Noetherian rings (II), J. Algebra 305 (2006), 974-992.

[4] C. Ciupercă, W. J. Heinzer, L. J. Ratliff, Jr., and D. E. Rush, Projectively full ideals in Noetherian rings, a survey, Algebra, Geometry and their Interactions Contemporary Mathematics, vol. 448, Amer. Math. Soc. 2007.

[5] O. Endler, Valuation Theory, Springer-Verlag, New York 1972.

[6] O. Endler, Finite separable field extensions with prescribed valuations, Manuscripta Math. 17 (1975), 383-408. 
[7] R. Gilmer, Prüfer domains and rings of integer-valued polynomials, J. Algebra, 129 (1990), 502-517.

[8] H. Hasse, Zwei Existensatz uber algebraische Zahlkorper, Math. Ann., 95 (1925), 229238.

[9] W. J. Heinzer, L. J. Ratliff, Jr., and D. E. Rush, Projectively full radical ideals in integral extension rings, J. Algebra, 317 (2007), 333-350.

[10] W. J. Heinzer, L. J. Ratliff, Jr., and D. E. Rush, Projective equivalence of ideals in Noetherian integral domains, J. Algebra, 320 (2008), 2349-2362.

[11] W. Krull, Über einen Existensatz der Bewertungstheorie, Abh. Math. Sem. Univ. Hamburg, 23 (1959), 29-55.

[12] M. Nagata, Local Rings, Interscience, John Wiley, New York, 1962.

[13] M. Nagata, Note on a paper of Samuel concerning asymptotic properties of ideals, Mem. Coll. Sci. Univ. Kyoto, Ser. A Math. 30 (1957), 165-175.

[14] D. Rees, Valuations associated with ideals (II), J. London Math. Soc. 36 (1956), 221228.

[15] P. Ribenboim, On the existence of fields with few discrete valuations, J. Reine Angew. Math. 216 (1964), 45-49.

[16] P. Samuel, Some asymptotic properties of powers of ideals, Annals of Math 56 (1952), $11-21$.

[17] I. Swanson and C. Huneke, Integral Closure of Ideals, Rings and Modules, Cambridge Univ. Press, Cambridge, 2006.

[18] R. Walker, Algebraic Curves, Springer-Verlag, New York, 1978.

[19] O. Zariski and P. Samuel, Commutative Algebra, Vol. 1, D. Van Nostrand, New York, 1958. 
Department of Mathematics, Purdue University, West Lafayette, Indiana 47907-1395 E-mail address: heinzer@math.purdue.edu

Department of Mathematics, University of California, Riverside, California 92521-0135 E-mail address: ratliff@math.ucr.edu

Department of Mathematics, University of California, Riverside, California 92521-0135 E-mail address: rush@math.ucr.edu 